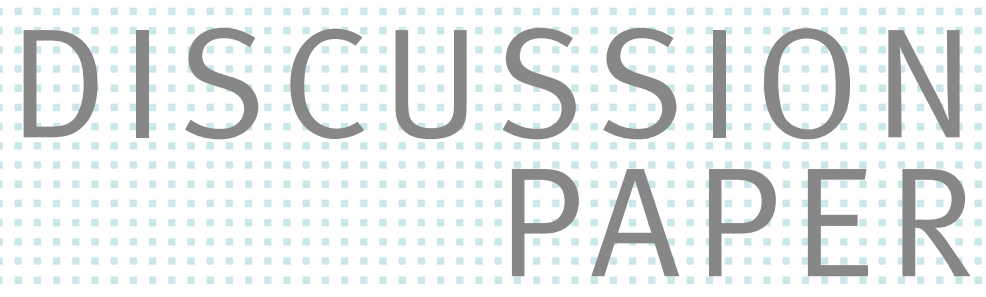

// NICOLAS FUGGER, PHILIPPE GILLEN, AND TOBIAS RIEHM

\title{
Procurement Design with Loss Averse Bidders
}




\title{
Procurement Design with Loss Averse
}

\section{Bidders}

\author{
Nicolas Fugger* ${ }^{*}$ Philippe Gillen ${ }^{\dagger} \quad$ Tobias RiehM $^{\ddagger}$
}

December 20, 2019

\begin{abstract}
We show that it is beneficial for a buyer to conduct a multi-stage mechanism if bidders are loss averse. In a first step, we derive a revenue equivalence principle. Fixing the multi-stage structure, the revenue is independent of the chosen payment rule. Secondly, we introduce a simple two-stage mechanism which always leads to a decrease in procurement costs compared to any single-stage auction. Finally we derive the optimal efficient two-stage mechanism.
\end{abstract}

JEL Classification: D44, D47, D90.

Keywords: Auctions; Experiment; Loss aversion; Preferences.

${ }^{*}$ ZEW. fugger@zew . de

${ }^{\dagger}$ ZEW. gillen@zew.de

${ }_{\text {ZZEW. riehm@zew.de }}$ 


\section{Contents}

\begin{tabular}{lll}
\hline & Introduction & 2
\end{tabular}

\begin{tabular}{|lll}
2 & Related Literature & 4
\end{tabular}

\begin{tabular}{lll}
\hline 3 & Model & 7
\end{tabular}

$3.1 \quad$ Equilibrium concept . . . . . . . . . . . . . . . . 8

3.2 Multi-stage mechanisms $\ldots \ldots \ldots \ldots \ldots$

$\begin{array}{lll}4 & \text { Analysis } & 10\end{array}$

4.1 Bidding behavior $\ldots \ldots \ldots \ldots \ldots \ldots$

$4.1 .1 \quad$ One-stage mechanisms $\ldots \ldots \ldots \ldots$

4.1 .2 Multi-stage mechanisms . . . . . . . . . . . . . . 11

$4.1 .3 \quad$ Example: First-price sealed-bid play-offs . . . . . . . . . . 16

4.2 Revenue equivalence principle . . . . . . . . . . . . . . . . . . . . . 19

$4.3 \quad$ A robust improvement over one-stage mechanisms . . . . . . . . . 21

4.4 Optimal efficient two-stage mechanism . . . . . . . . . . . . . 32

$\begin{array}{lll}5 \text { Conclusion } & 40\end{array}$

$\begin{array}{lll}6 & \text { Appendix } & 41\end{array}$

6.1 Mathematica code . . . . . . . . . . . . . . . 41 


\section{Introduction}

Procurement plays an important role both in the public and private sector. In Europe public procurement represented around $17 \%$ of the GDP in 2007 11 In many sectors of the industry the role of procurement is even more pronounced. The consulting company Oliver Wyman reports that suppliers are responsible for roughly $60 \%$ of the value added of a car ${ }^{2}$ Hence, small savings in average procurement costs translate to a substantial increase in overall profit margins.

In the past few decades reverse auctions have been established as one of the main tools to select suppliers and to determine prices in many industries. Depending on factors like size or complexity of a project, the procurement designer usually commits to a certain auction format. In the academic literature on auctions, it is typically assumed that the auction designer chooses between a first-price or secondprice payment rule and decides if she wants to conduct a static or dynamic auction. In the static formats, each bidder submits a sealed bid and the lowest bidder gets the contract. The dynamic formats typically considered are the Dutch auction and the English auction. In the English auction the price is decreased over time and bidders can drop out. It ends when the second-last bidder drops out. The winner is the last active bidder and he is paid the last displayed price. In the English auction the price increases over time and the first bidder who accepts the current price receives the contract and is paid the accepted price. In addition to the four auctions formats described, the auction designer could also determine the number of stages.

In single-stage auctions, suppliers hand in an offer once and the contract is allocated based on these offers. In multi-stage auctions, the first rounds are usually conducted to reduce the set of suppliers that can participate in the final round 3 Talks with practitioners suggest that especially in strategically important projects, multi-stage auctions are the preferred choice.

\footnotetext{
${ }^{1}$ Internal Market Scoreboard, ${ }^{o}$ 19, July 2009

2 https://www.oliverwyman.com/our-expertise/industries/automotive/ procurement.html

${ }^{3}$ Note that in these mechanisms, suppliers are typically restricted to hand in (weakly) more attractive offers in subsequent rounds.
} 
Interestingly, economic theory suggests that the use of multi-stage mechanisms cannot increase revenues above those that are achievable by one-stage mechanisms 4 when agents have standard preferences. However, if bidders are loss averse, the auction designer can increase her revenue by conducting multi-stage mechanisms. Proceeding to the next stage affects a bidder's winning probability and he therefore adjusts his reference point. The auction designer can exploit her influence on the bidders' reference points. Following Köszegi and Rabin (2006), we assume reference points are based on rational expectations $5^{5}$

A supplier who proceeds to the final stage of the multi-stage mechanism updates his winning probability. He knows that winning is now more likely than before. Loss aversion implies that such a bidder gets more attached to winning and is willing to make a more attractive offer, since losing in the final round would cause a high disutility. These additional gains and losses are anticipated by the agent before the auction and factored into his first-round bid. A straightforward way of implementing such a mechanism is by conducting a two-stage tournament. Suppliers compete in two semifinals and only the best supplier of each semifinal proceeds to the final stage 6

In line with von Wangenheim (2019), we assume that bidders evaluate outcomes in two dimensions, a money dimension and a good dimension 7 Consider a key account manager working for a supplier of a car manufacturer. When competing for a strategically important contract, he thinks in two independent dimensions: In the money domain, all monetary details such as his own costs, negotiated piece prices, investments etc. are captured. Independent of these details, the manager evaluates his chances of winning the contract and therefore getting a high level of

\footnotetext{
${ }^{4}$ We consider settings in which the time between the different stages is rather short and suppliers cannot adjust their product during the auction.

${ }^{5}$ There is an ongoing debate on how the reference point is formed. Some studies suggest that it is mainly driven by expectations, whereas others hold that it is mostly given by the status quo. For a discussion see Heffetz and List (2014) and references therein.

${ }^{6}$ If the number of suppliers is odd, one can conduct semifinals that are symmetric in expectation.

7 Lange and Ratan (2010) compare how the consideration of a one-dimensional reference point differs from the consideration of a two-dimensional reference point. They show that it can strongly affect predictions and argue that in most real world settings the consideration of a two-dimensional reference point is more reasonable.
} 
recognition within his company. If this is the case, the buyer of the car manufacturer could exploit this behavior when designing her procurement mechanism.

In this paper, we first derive a revenue equivalence principle for bidders that are loss averse in the good domain. For a fixed multi-stage structure, meaning which and how many bidders advance in the individual stages, the auctioneer's revenue is not dependent on the payment rule she chooses. This result considerably simplifies the analyses and allows us to concentrate on the structure of multi-stage mechanisms. Furthermore, as a side result, this entails that all single-stage static auctions lead to the same expected costs.

The main result of this paper is that the symmetric two-stage tournament always leads to a decrease in procurement costs compared to any (standard) singlestage auction. This result is robust, as it does not require knowledge about bidders' loss aversion. Hence, by conducting such a mechanism, the procurement designer's revenue strictly improves compared to all standard auctions if agents are loss averse, and makes no difference if not.

Finally, we derive the optimal efficient two-stage mechanism. When conducting two-stage mechanisms the procurement designer is confronted with a trade-off: On the one hand, she wants to maximize the attachment to winning the contract, and hence induce large winning probabilities to low-cost types. On the other hand, she can not neglect high-cost types, either. If high-cost types have an already very low chance of winning the project, they might insure themselves from a deviation from their expectation by bidding even lower. Taking into account the bidders' degree of loss aversion, the optimal mechanism thus creates the level of uncertainty that optimally solves this trade-off.

\section{Related Literature}

Our paper contributes to the literature on expectations-based loss aversion. The concept of loss aversion has been studied since the seminal paper of Kahneman, Knetsch, and Thaler (1990). In their paper, they introduce the endowment effect and experimentally show that a subjects' valuation for a certain good increases 
when they are physically endowed with the good. According to this strand of literature subjects have a reference point and a deviation from this reference point in direction of losses has a larger impact on utility than a deviation in direction of gains.

A discussion around the formation of these reference points has risen in the literature. Köszegi and Rabin (2006) suggest that the reference point is based on rational expectations. In an auction, this means that bidders have a certain probability of winning in mind and feel losses and gains compared to these expectations. As a consequence, a bidder expecting to win a good with a high probability suffers more from not winning than if he gauged his chances of winning as slim.

Our paper is most closely related to von Wangenheim (2019), who compares a sealed-bid second-price auction to an English auction assuming that bidders are loss averse and that their reference point is given by rational expectations. While both formats are strategically equivalent in independent private value settings if bidders have standard preferences, he shows that the second-price sealed-bid auction dominates the English auction if bidders are loss averse. The intuition is as follows: At the beginning of the English auction a bidder has the same chance of winning as in the second-price sealed-bid auction. However, during the course of the English auction the winning probability decreases and the bidder becomes less attached to the good. As a consequence, his willingness to pay decreases and he will drop out before the price is reached that he would have bid in the second-price sealed-bid auction.

Similar to von Wangenheim (2019), Ehrhart and Ott (2014) compare two standard auction formats for bidders with reference-dependent preferences. Comparing the Dutch auction to the English auction they show that the Dutch auction outperforms the English auction. The intuition is closely related to von Wangenheim (2019) and to our paper. For a given valuation a bidder has the same winning probability at the beginning of the Dutch auction and the English auction. However, while the winning probability decreases during the course of the English auction, it increases during the course of the Dutch auction. Hence, the attachment to the good is larger in the Dutch auction and bidders are thus willing to bid more ag- 
gressively. Similarly, a bidder who advances a stage in our setting also updates his winning probability and therefore his attachment to the good increases. This, in return, increases the bid he is willing to submit.

Banerji and Gupta (2014) and Rosato and Tymula (2019) provide evidence for the effect of expectations-based loss aversion in auction environments. In a setting in which participants compete in a second-price auction for a real good, they observe that bidders bid less when their winning probability was smaller. This observation stands in contrast to the predictions of standard theory which implies that subjects have a dominant strategy of bidding their true valuation independent of their winning probability. In contrast to that, loss aversion implies that a bidder with a higher chance of winning is more attached to the good and, hence, willing to bid more.

In contrast to the existing paper on auctions with loss averse bidders, we do not concentrate on comparing standard auction formats but investigate the following question: How can an auctions designer exploit bidders' loss aversion to increase her revenue?

Given this research question our work is also related to Maskin and Riley (1984) who also investigate how the auction designer can increase her revenue if bidders have a behavioral bias, in their case risk aversion. Similar to us, they present an optimal mechanism that needs to be fine-tuned to bidders' risk preferences and seems too complex to be implemented in practice. While our management implication is that simple two-stage mechanisms outperform one-stage auctions if bidders are loss averse, they show that first-price auctions outperform second-price auctions if bidders are risk averse.

Another related paper is Engelbrecht-Wiggans and Katok (2007). They analyze how the auction designer can exploit regret aversion of bidders. They show that the right information design, namely revealing the best bid but concealing all other bids, allows the auction designer to increase her revenue. 


\section{Model}

In this section, we introduce the formal model. We consider $n \geq 2$ ex-ante symmetric bidders competing for one indivisible good. The value $v_{i}$ of bidder $i \in\{1, \ldots, n\}$ for the good is privately drawn from a distribution $F, v_{i} \stackrel{\text { iid. }}{\sim} F[0,1] . F$ is assumed to have a differentiable density $f$ which is strictly positive on its support $[0,1]$. Moreover, $F$ is common knowledge. Bids are placed after learning the value for the good.

For loss aversion we follow Köszegi and Rabin (2006). We assume that bidders are loss averse in the good domain $g$ representing the item the winner of the auction receives $8^{8}$ Furthermore, we assume bidders to be narrow-bracketers, following the definition of von Wangenheim (2019). Let $x^{m}$ be the price a bidder pays if he wins and $x^{g}$ a binary variable that is equal to one if the bidder wins the good and zero else. For an outcome $x=\left(x^{c}, x^{g}\right)$, valuation $v$ for the good, and the reference consumption $r^{g} \in\{0,1\}$, agent's utility is given by

$$
u\left(x \mid r^{g}\right)=x^{c}+v x^{g}+\mu^{g}\left(v x^{g}-v r^{g}\right) .
$$

Following Kőszegi and Rabin (2006), we assume $\mu^{g}$ to be a piecewise linear function with a kink at zero,

$$
\mu^{g}(y)= \begin{cases}\eta^{g} y & \text { if } y \geq 0 \\ \lambda^{g} \eta^{g} y & \text { if } y<0 .\end{cases}
$$

Here $\mu^{g}$ denotes the gain-loss utilities in the good dimension, where $\eta^{g}>0$ and $\lambda^{g}>1$. We assume non-dominance-of-gain-loss-utility, which means for a multistage mechanism with $k$ stages $\eta^{g}\left(\lambda^{g}-1\right) \leq 1 / k !^{9}$ The importance of the nondominance-of-gain-loss-utility bounds on $\eta^{i}$ and $\lambda^{i}$ are laid out in Herweg, Müller, and Weinschenk (2010). To summarize, if $\eta^{g}\left(\lambda^{g}-1\right)>1 / k$, a decision maker might choose stochastically dominated choices because he ex-ante expects to experience a net loss. For example, such a decision maker might choose a payment of zero over

\footnotetext{
${ }^{8} \mathrm{We}$ assume that bidders are not loss averse in the money domain. This assumption is in line with Horowitz and McConnell (2003), who argue that the endowment effect is "highest for non-market goods, next highest for ordinary private goods, and lowest for experiments involving forms of money."

${ }^{9}$ This bound for non-dominance-of-gain-loss-utility is derived in Section 4.1 .2
} 
a lottery with slim chances of winning a strictly positive amount of money to avoid the disappointment, should he lose.

The interpretation of this gain-loss utility is that bidders perceive, in addition to their classical utility, a feeling of gain or loss, depending on the deviation from their reference consumption.

The reference point in our paper is assumed to be determined by rational expectations following Köszegi and Rabin (2006).

\subsection{Equilibrium concept}

Following von Wangenheim (2019), we adapt Köszegi and Rabin (2006)'s equilibrium concept under uncertainty, according to which bidders form their strategy after learning their valuation. We apply the concept of unacclimated personal equilibria, which is, as argued by Köszegi and Rabin (2006), the appropriate concept in auction settings. Fixing the opponents' strategies, let $H\left(b, v_{i}\right)$ denote $i$ 's payoff distribution given his draw $v_{i}$ from a continuous distribution $F(v)$ and his bid $b$. A bid $b^{*}$ constitutes an unacclimated personal equilibrium (UPE), if for all $b$

$$
U\left[H\left(b^{*}, v_{i}\right) \mid H\left(b^{*}, v_{i}\right)\right] \geq U\left[H\left(b, v_{i}\right) \mid H\left(b^{*}, v_{i}\right)\right] .
$$

That means, given your reference point is determined by the payoff distribution resulting from an (exogenous) bid $b^{*}$, it is a best response to bid $b^{*}$.

\subsection{Multi-stage mechanisms}

In a multi-stage mechanism, bidders participate in $k$ stages and submit a bid in each one of them. The rules of the mechanism include how many stages there are and which bidders advance to the next stage. Bids are binding and cannot be lowered in subsequent stages.

As an example, consider four bidders and a mechanism with two stages. In the first stage, the semi-final, all four bidders submit an offer. The two bidders with the highest offers then advance to the final, where they submit another offer. The highest offer in the final is then the winner of the auction 10

\footnotetext{
${ }^{10}$ We call this mechanism the "play-offs", it is analysed in section subsubsection 4.1.3
} 
In this section, we introduce the formal notation for multi-stage mechanisms. To completely characterize a multi-stage structure, we need to define the number of stages $k$ and for each of the $k$ stages, which of the bidders advance to the next stage. For $N$ bidders, let $\mathfrak{B}=\left\{{ }^{j} B_{1},{ }^{j} B_{2}, \ldots,{ }^{j} B_{N}\right\}$ be the set of bids for each bidder in a stage $j \leq k$. We restrict ourselves to multi-stage mechanisms that are symmetric in expectation. This means that in some stage $j$ of the mechanism, each bidder has the same expectation of number of opponents he is facing even if there are asymmetric groups $\$$ Borrowing from order statistics notation, a multi-stage mechanism is then defined by $(\boldsymbol{\mu}, \mathfrak{M})$, with $\boldsymbol{\mu}$ the payment rule and

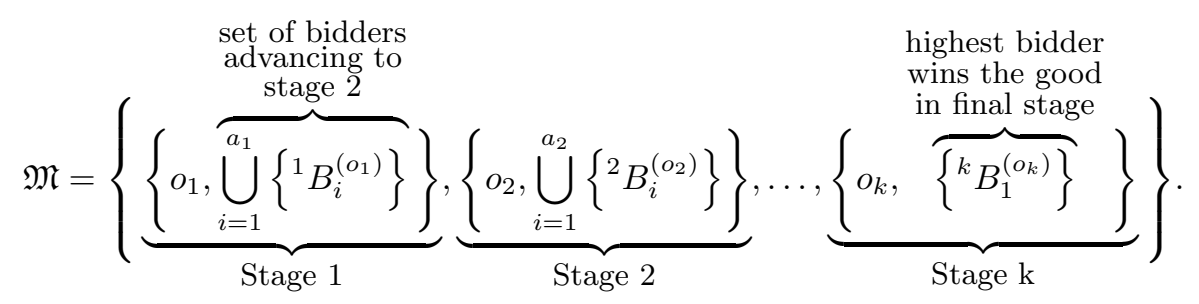

Here the $o_{j}$ are the number of bidders per subgroup in stage $j$ and $a_{j}$ the number of bidders advancing from stage $j$ to $j+1\left[12\right.$ It must hold that $a_{i} \leq o_{i}$ and $o_{j} \leq N$ where $N$ is the total number of bidders.

\footnotetext{
${ }^{11}$ If there are asymmetric groups, the probability of being matched to a specific group has to be stated.

${ }^{12}$ This implies that only the highest $o_{j+1}$ bidders of each subgroup advance from stage $j$ to $j+1$.
} 


\section{Analysis}

The theory section is structured as follows. In Section 4.1 we derive general properties of the equilibrium bidding behavior in one- and multi-stage mechanisms. In Section 4.2 we show that fixing the multi-stage structure implies a revenue equivalence principle: the chosen payment rule does not affect the expected revenue of a mechanism. We then present a robust, easily implementable improvement over onestage mechanisms in Section 4.3 and finally derive the optimal efficient two-stage mechanism in Section 4.4

\subsection{Bidding behavior}

\subsubsection{One-stage mechanisms}

Assume that the bidders have standard preferences and let bidders participate in a standard auction $A[13$ Further assume that the other bidders bid according to an increasing and absolutely continuous bidding function $\beta^{A}$. The payment rule of the auction is denoted by $\boldsymbol{\mu}^{A}\left(b_{i}, b_{-i}\right)$ and the expected payment by $m^{A}\left(b_{i}\right)$. Define $G(b):=F_{1}^{(N-1)} \circ \beta^{A^{-1}}(b)$ the winning probability with a bid $b$ in the auction. Then the expected utility of bidder $i$ having value $v_{i}$ and bidding $b$ is given by

$$
u_{i}^{A}\left(v_{i}, b\right)=G(b) v-m^{A}(b) .
$$

We now introduce loss aversion with bidders being loss averse only in the good domain. Given a reference bid $b^{*}$, the expected utility is then given by

$$
\begin{aligned}
\multicolumn{1}{l}{u_{i}^{A}\left(v_{i}, b \mid b^{*}\right)=} & G(b) v-m^{A}(b) \\
& +G(b)\left(1-G\left(b^{*}\right)\right) \mu^{g}(v-0) \\
\text { feeling of gain, good domain } & +(1-G(b)) G\left(b^{*}\right) \mu^{g}(0-v) \\
= & G(b) v-m^{A}(b) \\
\text { feeling of loss, good domain } & +G(b)\left(1-G\left(b^{*}\right)\right) \eta^{g} v \\
& +(1-G(b)) G\left(b^{*}\right) \eta^{g} \lambda^{g}(-v)
\end{aligned}
$$

\footnotetext{
$\sqrt[13]{\text { Krishna }}$ (2009) defines a standard auction as an auction where the person who bids the highest amount is awarded the object.
} 
Bidders optimize $u_{i}^{A}$ with respect to $b$.

\subsubsection{Multi-stage mechanisms}

As a first step, we show that in equilibrium, bidders submit the same bid in every stage of the mechanism if non-dominance-of-gain-loss-utility holds.

Proposition 1. In a multi-stage mechanism, bidders submit the same bid in every stage.

Consider bidder $i$. Assume the other bidders bid according to an increasing, absolutely continuous bidding function $\beta_{j}^{M S}$, where $j$ denotes the stage. The structure of the multi-stage mechanism, i.e. how many bidders advance in the individual stages and how many opponents they face in each stage, is then encoded in the probabilities to reach the individual stages of the mechanism. Let $\phi_{j}$ be defined such that $\phi_{j} \circ F \circ \beta_{j}^{M S^{-1}}$ is the probability of reaching stage $j+1$ given the bidder reached stage $j$. ${ }^{14}$ Let $\vec{b}=\left(b_{1}, b_{2}, \ldots, b_{k}\right)$ be the vector of bids of bidder 1 . This means that the ex-ante probability to win the auction is given by

$$
\operatorname{Prob}^{\text {ex-ante }}(\text { win with } b)=\prod_{j=1}^{k} \phi_{j}\left(b_{j}\right)=: H(\vec{b}) \text {. }
$$

Note that to simplify the notation, we define that advancing to stage $k+1$ means winning the auction.

It is useful to define the probability to reach stage $l$, given that the bidder reached stage $i$. Let $i<l$. Then $\Phi_{i}^{l}$ is given by

$$
\begin{aligned}
& \operatorname{Prob}(\text { get to } l \text { with } \vec{b} \mid \text { get to } i \text { with } \vec{b}) \\
= & \frac{\operatorname{Prob}(\text { get to } l \text { with } \vec{b} \& \text { get to } i \text { with } \vec{b})}{\operatorname{Prob}(\text { get to } i \text { with } \vec{b})} \\
= & \frac{\operatorname{Prob}(\text { get to } l \text { with } \vec{b})}{\operatorname{Prob}(\text { get to } i \text { with } \vec{b})}=\prod_{j=i}^{l-1} \phi_{j}\left(b_{j}\right):=\Phi_{i}^{l}(\vec{b})
\end{aligned}
$$

\footnotetext{
${ }^{14} \mathrm{The} \phi_{j} \circ F$ are expressions of probability and thus inherit the properties of the original distribution functions.
} 
The probability to win the auction given the bidder reached stage $i$ is given by

$$
\operatorname{Prob}(\text { win with } \vec{b} \mid \text { reached } i)=\Phi_{i}^{k+1}(\vec{b})
$$

For each stage $l$, given a reference bid $b_{l}^{*}$, the bidder experiences a gain-loss utility in expectation. On one hand, the bidder might win with his bid $b_{l}$ but has expected to lose with the reference bid $b_{l}^{*}$. He then experiences a gain in the good domain with respect to the reference outcome. On the other hand, the bidder might lose in one of the stages with his bid $b_{l}$ but has expected to win with the reference bid $b_{l}^{*}$. He then experiences a loss in the good domain. This holds true for every stage.

Consider a standard auction based payment rule, $\boldsymbol{\mu}^{M S}$. The expected payment of the multi-stage mechanism composed by the expected amount a bidder has to pay and the probability of him having to pay it,

$$
\begin{aligned}
m^{M S}(\vec{b}) & =\text { Prob }(\text { having to pay with } \vec{b}) \mathbb{E}\left[\boldsymbol{\mu}^{M S} \mid \vec{b}, \vec{b}_{-i}\right] \\
& =: P^{\text {pay }}(\vec{b}) \mathbb{E}\left[\boldsymbol{\mu}^{M S} \mid \vec{b}, \vec{b}_{-i}\right]
\end{aligned}
$$

For the first-, second-, ...-price auction, we have $P^{\text {pay }}(\vec{b})=H(\vec{b})$, while for the all-pay auction we have that $P^{\text {pay }}(\vec{b})=1$. Generally, $P^{\text {pay }}(\vec{b})$ either depends linearly on the $\phi_{i}$ for $i \in\{1, \ldots, k\}$ or is constant 15 This means that it holds for all $j<k$,

$$
\frac{\partial m^{M S}(\vec{b})}{\partial\left(\phi_{j}(\vec{b})\right)} \leq \frac{m^{M S}(\vec{b})}{\phi_{j}(\vec{b})} .
$$

Combining the results from above, we arrive at the following utility function for

\footnotetext{
${ }^{15}$ The fringe case where $\mathbb{E}\left[\boldsymbol{\mu}^{M S} \mid \vec{b}, \vec{b}_{-i}\right]$ consists of a lottery that explicitly depends on a $\phi_{i}$ with $i \in\{1, \ldots, k-1\}$ is excluded here. The lottery may depend on $\vec{b}$.
} 
loss averse bidders in multi-stage mechanisms,

$$
\begin{aligned}
u^{M S}\left(v_{i}, \vec{b} \mid \vec{b}^{*}\right)= & H(\vec{b}) v_{i}-m^{M S}(\vec{b}) \\
& +\underbrace{\sum_{i=1}^{k} \Phi_{1}^{k+1}(\vec{b})\left(1-\Phi_{i}^{i+1}\left(\vec{b}^{*}\right)\right) \mu^{g}(v-0)}_{\text {expecting to win with } \vec{b}, \text { to lose with } \vec{b}^{*}} \\
& +\underbrace{\sum_{i=1}^{k} \Phi_{0}^{i}(\vec{b})\left(1-\Phi_{i}^{i+1}(\vec{b})\right) \Phi_{i}^{k+1}\left(\vec{b}^{*}\right) \mu^{g}(0-v)}_{\text {expecting to lose with } \vec{b}, \text { to win with } \vec{b}^{*}} \\
= & H(\vec{b}) v_{i}-m^{M S}(\vec{b}) \\
& +\sum_{i=1}^{k} \Phi_{1}^{k+1}(\vec{b})\left(1-\Phi_{i}^{i+1}\left(\vec{b}^{*}\right)\right) \eta^{g} v \\
& +\sum_{i=1}^{k}\left(\Phi_{0}^{i}(\vec{b})-\Phi_{0}^{i+1}(\vec{b})\right) \Phi_{i}^{k+1}\left(\vec{b}^{*}\right) \eta^{g} \lambda^{g}(-v) .
\end{aligned}
$$

Note that we can bound $m^{M S}$ from above depending on $v_{i}$ and $\vec{b}^{*}$ since a bidder will not submit bids that result in a negative expected utility,

$$
\begin{aligned}
u^{M S}\left(v_{i}, \vec{b} \mid \vec{b}^{*}\right) \stackrel{!}{>} & 0 \\
\Rightarrow m^{M S}(\vec{b}) \stackrel{!}{<} & H(\vec{b}) v_{i} \\
& +\sum_{i=1}^{k} \Phi_{1}^{k+1}(\vec{b})\left(1-\Phi_{i}^{i+1}\left(\vec{b}^{*}\right)\right) \eta^{g} v \\
& +\sum_{i=1}^{k} \Phi_{0}^{i}(\vec{b})\left(1-\Phi_{i}^{i+1}(\vec{b})\right) \Phi_{i}^{k+1}\left(\vec{b}^{*}\right) \eta^{g} \lambda^{g}(-v) .
\end{aligned}
$$

Also note that the right-hand side does not contain $b_{j}$ outside of $\phi_{j}$ for all $j \in$ $\{1, \ldots, k-1\}$. This means that for the first $k-1$ stages, a bidder can directly optimize over the probability of advancing to the next stage instead of optimizing over the bids that induce probabilities. Our equilibrium concept is UPE, this implies 
that the first-order condition for $i \in\{1, \ldots, k-1\}$, is given by

$$
\begin{aligned}
& \left.\frac{\partial u^{M S}\left(v_{i}, \vec{b} \mid \vec{b}^{*}\right)}{\partial\left(\phi_{i}\left(b_{i}\right)\right)}\right|_{\vec{b}^{*}=\vec{b}}=\frac{\prod_{j=1}^{k} \phi_{j}\left(b_{j}\right)}{\phi_{i}\left(b_{i}\right)} v_{i}-\frac{\partial m^{M S}(\vec{b})}{\partial\left(\phi_{i}\left(b_{i}\right)\right)} \\
& \quad+\left.\frac{\partial}{\partial\left(\phi_{i}\left(b_{i}\right)\right)} \sum_{l=1}^{k} \prod_{j=1}^{k} \phi_{j}\left(b_{j}\right)\left(1-\Phi_{l}^{l+1}\left(\vec{b}^{*}\right)\right) \eta^{g} v_{i}\right|_{\vec{b}^{*}=\vec{b}} \\
& \quad+\left.\frac{\partial}{\partial\left(\phi_{i}\left(b_{i}\right)\right)} \sum_{l=1}^{k} \prod_{j=0}^{l-1} \phi_{j}\left(b_{j}\right) \Phi_{l}^{k+1}\left(\vec{b}^{*}\right) \eta^{g} \lambda^{g}\left(-v_{i}\right)\right|_{\vec{b}^{*}=\vec{b}} \\
& \quad-\left.\frac{\partial}{\partial\left(\phi_{i}\left(b_{i}\right)\right)} \sum_{l=1}^{k} \prod_{j=0}^{l} \phi_{j}\left(b_{j}\right) \Phi_{l}^{k+1}\left(\vec{b}^{*}\right) \eta^{g} \lambda^{g}\left(-v_{i}\right)\right|_{\vec{b}^{*}=\vec{b}} .
\end{aligned}
$$

We now rearrange the terms. 201 simplifies to

$$
\sum_{l=1}^{k} \frac{\prod_{j=1}^{k} \phi_{j}\left(b_{j}\right)}{\phi_{i}\left(b_{i}\right)}\left(1-\phi_{l}\left(b_{l}\right)\right) \eta^{g} v_{i}
$$

For 21], we get

$$
\begin{aligned}
\sum_{l=i+1}^{k} \frac{\prod_{j=1}^{l-1} \phi_{j}\left(b_{j}\right)}{\phi_{i}\left(b_{i}\right)} \prod_{j=l}^{k} \phi_{j}\left(b_{j}\right) \eta^{g} \lambda^{g}\left(-v_{i}\right) & =\sum_{l=i+1}^{k} \frac{\prod_{j=1}^{k} \phi_{j}\left(b_{j}\right)}{\phi_{i}\left(b_{i}\right)} \eta^{g} \lambda^{g}\left(-v_{i}\right) \\
& =\frac{\prod_{j=1}^{k} \phi_{j}\left(b_{j}\right)}{\phi_{i}\left(b_{i}\right)} \eta^{g} \lambda^{g}\left(-v_{i}\right)(k-i) .
\end{aligned}
$$

For 221 , we get

$$
-\sum_{l=i}^{k} \frac{\prod_{j=1}^{l} \phi_{j}\left(b_{j}\right)}{\phi_{i}\left(b_{i}\right)} \prod_{j=l}^{k} \phi_{j}\left(b_{j}\right) \eta^{g} \lambda^{g}\left(-v_{i}\right)=-\sum_{l=i}^{k} \frac{\prod_{j=1}^{k} \phi_{j}\left(b_{j}\right)}{\phi_{i}\left(b_{i}\right)} \phi_{l}\left(b_{l}\right) \eta^{g} \lambda^{g}\left(-v_{i}\right) .
$$

Define

$$
\alpha:=\frac{\prod_{j=1}^{k} \phi_{j}\left(b_{j}\right)}{\phi_{i}\left(b_{i}\right)} .
$$


We arrive at

$$
\begin{aligned}
& \left.\frac{\partial u^{M S}\left(v_{i}, \vec{b} \mid \vec{b}^{*}\right)}{\partial\left(\phi_{i}\left(b_{i}\right)\right)}\right|_{\vec{b}^{*}=\vec{b}}=-\frac{\partial m^{M S}(\vec{b})}{\partial\left(\phi_{i}\left(b_{i}\right)\right)} \\
& +\alpha v_{i}+\eta^{g} v_{i} \alpha \sum_{l=1}^{k}\left(1-\phi_{l}\left(b_{l}\right)\right) \\
& -\eta^{g} \lambda^{g} v_{i} \alpha(k-i)+\eta^{g} \lambda^{g} v_{i} \sum_{l=i}^{k} \phi_{l}\left(b_{l}\right) \\
& \geq-\frac{m^{M S}(\vec{b})}{\phi_{i}\left(b_{i}\right)}+\alpha v_{i}+\eta^{g} v_{i} \alpha \sum_{l=1}^{k}\left(1-\phi_{l}\left(b_{l}\right)\right) \\
& -\eta^{g} \lambda^{g} v_{i} \alpha(k-i)+\eta^{g} \lambda^{g} v_{i} \sum_{l=i}^{k} \phi_{l}\left(b_{l}\right) \\
& \geq-\left[H(\vec{b}) v_{i}+\sum_{i=1}^{k} \Phi_{1}^{k+1}(\vec{b})\left(1-\Phi_{i}^{i+1}\left(\vec{b}^{*}\right)\right) \eta^{g} v_{i}\right. \\
& \left.+\sum_{i=1}^{k} \Phi_{0}^{i}(\vec{b})\left(1-\Phi_{i}^{i+1}(\vec{b})\right) \Phi_{i}^{k+1}\left(\vec{b}^{*}\right) \eta^{g} \lambda^{g}\left(-v_{i}\right)\right]_{\vec{b}^{*}=\vec{b}} \\
& +\alpha v_{i}+\eta^{g} v_{i} \alpha \sum_{l-1}^{k}\left(1-\phi_{l}\left(b_{l}\right)\right) \\
& -\eta^{g} \lambda^{g} v_{i} \alpha(k-i)+\eta^{g} \lambda^{g} v_{i} \sum_{l=i}^{k} \phi_{l}\left(b_{l}\right) \\
& =-\alpha v_{i}+\eta^{g}\left(\lambda^{g}-1\right) v_{i} \alpha \sum_{l=1}^{k}\left(1-\phi_{l}\left(b_{l}\right)\right) \\
& +\alpha v_{i}+\eta^{g} v_{i} \alpha \sum_{l-1}^{k}\left(1-\phi_{l}\left(b_{l}\right)\right) \\
& -\eta^{g} \lambda^{g} v_{i} \alpha(k-i)+\eta^{g} \lambda^{g} v_{i} \sum_{l=i}^{k} \phi_{l}\left(b_{l}\right) \\
& =\eta^{g} \lambda^{g} v_{i} \alpha\left(i-\sum_{l=1}^{i-1} \phi_{l}\right)>0 .
\end{aligned}
$$

Note that we need to make sure that the expression in the brackets in step 30 is 
positive for all $\phi_{j}$. This means it needs to hold that

$$
\begin{aligned}
\alpha v_{i}-\eta^{g}\left(\lambda^{g}-1\right) v_{i} \alpha \sum_{l=1}^{k}\left(1-\phi_{l}\left(b_{l}\right)\right) & \stackrel{!}{\geq} 0 \\
\Leftrightarrow & -\eta^{g}\left(\lambda^{g}-1\right) \geq \frac{1}{\sum_{l=1}^{k}\left(1-\phi_{l}\left(b_{l}\right)\right)} \\
& \Leftrightarrow \eta^{g}\left(\lambda^{g}-1\right) \stackrel{!}{\leq} \frac{1}{\sum_{l=1}^{k}\left(1-\phi_{l}\left(b_{l}\right)\right)} \\
& \Leftrightarrow \eta^{g}\left(\lambda^{g}-1\right) \stackrel{!}{\leq} \min _{\phi} \frac{1}{\sum_{l=1}^{k}\left(1-\phi_{l}\left(b_{l}\right)\right)} \\
& \Rightarrow \eta^{g}\left(\lambda^{g}-1\right) \stackrel{!}{\leq} \frac{1}{k} .
\end{aligned}
$$

For every stage, a bidder experiences gain-loss utility. All-in-all, this means that the non-dominance of gain-loss utility has to hold for every stage, in total $\eta^{g}\left(\lambda^{g}-1\right) \stackrel{!}{\leq}$ $\frac{1}{k}$.

Interpreting $\phi_{j}$ as the distribution of bids that a bidder needs to beat in expectation to order to advance to stage $j+1, \sqrt{32}$ implies that a bidder will always want to induce the highest possible probability to advance to the final stage with his bid $\vec{b}$. This implies that a bidder will cap his bids in stages 1 to $k-1$ by the bid he submits in the final, pay-off relevant stage. A bidder therefore optimizes

$$
\begin{aligned}
u^{M S}\left(v_{i}, b \mid b^{*}\right)= & G(b) v_{i}-m^{M S}(b) \\
& +\sum_{i=1}^{k} \Phi_{1}^{k+1}(b)\left(1-\Phi_{i}^{i+1}\left(b^{*}\right)\right) \eta^{g} v \\
& +\sum_{i=1}^{k}\left(\Phi_{0}^{i}(b)-\Phi_{0}^{i+1}(b)\right) \Phi_{i}^{k+1}\left(b^{*}\right) \eta^{g} \lambda^{g}(-v)
\end{aligned}
$$

over $b$.

\subsubsection{Example: First-price sealed-bid play-offs}

To get an idea what such a multi-stage mechanism can look like and of how to apply what we've derived so far, let us take a look at the following multi-stage mechanism 


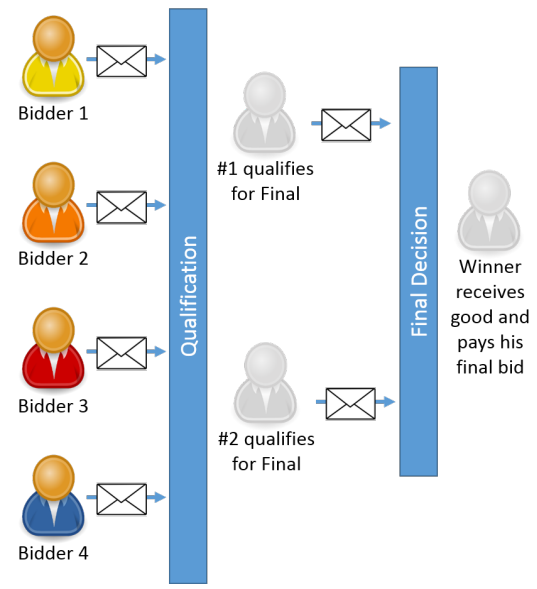

Figure 1: The first-price sealed-bid play-offs.

with four bidders. As can be seen in Figure 1, the FPSB play-offs consists of 2 stages.

1. Out of the four bidders, the two highest bidders are advancing to the second stage.

2. Out of the two remaining bidders, the highest bid wins.

We can write $\mathfrak{M}^{P O}$ as

$$
\mathfrak{M}^{P O}=\{\underbrace{\left\{4,\left\{B_{1}^{(4)}, B_{2}^{(4)}\right\}\right\}}_{\text {Stage } 1}, \underbrace{\left\{2,\left\{B_{1}^{(2)}\right\}\right\}}_{\text {Stage } 2}\} .
$$

The payment rule $\boldsymbol{\mu}$ is given by the first-price auction payment rule. With proposition 1, we can assume bidders to bid the same in every stage. Assume the other bidders bid according to an increasing, absolutely continuous bidding function $\beta^{P}$. In the first stage, bidder $i$ advances if he beats at least the second highest opponent. This yields

$$
\phi_{1} \circ F=F_{2}^{(3)}=3 F^{2}-2 F^{3} .
$$


Given that the bidder reached stage two, the bidder wins if he beats his strongest opponent,

$$
\begin{aligned}
\phi_{2} \circ F \circ \beta^{P^{-1}}(b) & =\operatorname{Prob}\left(b>\beta^{P}\left(v_{1}^{(3)}\right) \mid b>\beta^{P}\left(v_{2}^{(3)}\right)\right) \\
& =\frac{F\left(\beta^{P^{-1}}(b)\right)^{3}}{3 F\left(\beta^{P^{-1}}(b)\right)^{2}-2 F\left(\beta^{P^{-1}}(b)\right)^{3}} .
\end{aligned}
$$

The underlying auction format is the first-price auction, the expected payment is given by $m^{T}(b)=G(b) b$. The utility is then given by

$$
\begin{aligned}
& u^{P}\left(v_{i}, b \mid b^{*}\right)=G\left(b\left(v_{i}-b\right)\right. \\
& \underbrace{+F_{1}^{(3)}\left(\beta^{P^{-1}}(b)\right)\left(1-F_{2}^{(3)}\left(\beta^{P^{-1}}\left(b^{*}\right)\right)\right) \eta^{g} v}_{\text {win but would have lost in stage 1 with } b^{*}} \\
& +\underbrace{+F_{1}^{(3)}\left(\beta^{P^{-1}}(b)\right)\left(1-\frac{F_{1}^{(3)}\left(\beta^{P^{-1}}\left(b^{*}\right)\right)}{F_{2}^{(3)}\left(\beta^{P^{-1}}\left(b^{*}\right)\right)}\right) \eta^{g} v}_{\text {win but would have lost in stage 2 with } b^{*}} \\
& \underbrace{\left.+1-F_{2}^{(3)}\left(\beta^{P^{-1}}(b)\right)\right) F_{1}^{(3)}\left(\beta^{P^{-1}}\left(b^{*}\right)\right) \eta^{g} \lambda(-v)}_{\text {don't advance to 2nd stage but would have won with } b^{*}} \\
& +\underbrace{+F_{2}^{(3)}\left(\beta^{P^{-1}}(b)\right)\left(1-\frac{F_{1}^{(3)}\left(\beta^{P^{-1}}(b)\right)}{F_{2}^{(3)}\left(\beta^{P^{-1}}(b)\right)}\right) \frac{F_{1}^{(3)}\left(\beta^{P^{-1}}\left(b^{*}\right)\right)}{F_{2}^{(3)}\left(\beta^{P^{-1}}\left(b^{*}\right)\right)} \eta^{g} \lambda(-v)}_{\text {get to 2nd stage \& lose but would have won with } b^{*}} .
\end{aligned}
$$

We are interested in finding the equilibrium bidding function for this multi-stage auction. Our equilibrium concept is UPE, this implies that the first-order condition is given by

$$
\left.\left(\frac{\partial u^{P}\left(v_{i}, b \mid b^{*}\right)}{\partial b}\right)\right|_{b^{*}=\beta^{P}\left(v_{i}\right)} \stackrel{!}{=} 0 .
$$

In equilibrium it holds that $b=\beta^{P}\left(v_{i}\right)$. To simplify notation, let $F_{m}^{(3)}=: F_{m}$. The resulting ordinary differential equation admits a closed form solution,

$$
\begin{aligned}
\beta^{P}\left(v_{i}\right)=\frac{1}{F_{1}\left(v_{i}\right)} \int_{0}^{v_{i}} s\left(f_{1}(s)\right. & +\eta^{g} \lambda^{g}\left(f_{2}(s) F_{1}(s)-\left(f_{2}(s)-f_{1}(s)\right) \frac{F_{1}(s)}{F_{2}(s)}\right) \\
& \left.+\eta^{g} f_{1}(s)\left(2-\frac{F_{1}(s)}{F_{2}(s)}-F_{2}(s)\right)\right) d s .
\end{aligned}
$$




\subsection{Revenue equivalence principle}

In this section, we show that once we fix the multi-stage structure of the procurement mechanism, a revenue equivalence principle holds. This means that an auctioneer does not need to worry about the payment rule of her mechanism ${ }^{16}$

Proposition 2 (Revenue equivalence principle for loss averse bidders). Suppose that values are independently and identically distributed and that bidders are loss averse in the good domain. Fix the multi-stage structure $\mathfrak{M}$. For every standard auction payment rule $\boldsymbol{\mu}$, any symmetric and increasing equilibrium such that the expected payment of a bidder with value zero is zero, yields the same expected revenue to the seller.

Consider multi-stage mechanism $M S=(\boldsymbol{\mu}, \mathfrak{M})$, with $\boldsymbol{\mu}$ some standard auction payment rule, and fix a symmetric, strictly increasing equilibrium bidding function $\beta^{M S}$. Let $m^{M S}\left(v_{i}\right)$ be the equilibrium expected payment in the mechanism by bidder $i$ with value $v_{i}$. Suppose that $\beta^{M S}$ is such that $m^{M S}(0)=0$. Define the ex-ante expected gain-loss utility in the good domain $\Theta^{g}$ such that

$$
\begin{aligned}
\Theta^{g}\left(b \mid b^{*}\right):= & \sum_{i=1}^{k} \Phi_{1}^{k+1}(b)\left(1-\Phi_{i}^{i+1}\left(b^{*}\right)\right) \eta^{g} v \\
& +\sum_{i=1}^{k}\left(\Phi_{0}^{i}(b)-\Phi_{0}^{i+1}(b)\right) \Phi_{i}^{k+1}\left(b^{*}\right) \eta^{g} \lambda^{g}(-v),
\end{aligned}
$$

yielding

$$
u^{M S}\left(v_{i}, b \mid b^{*}\right)=G(b) v_{i}-m^{M S}(b)+\Theta^{g}\left(b \mid b^{*}\right) .
$$

Consider bidder $i$ and suppose other bidders are following the equilibrium strategy $\beta^{M S}$. Consider the expected payoff of bidder $i$ with value $v_{i}$ deviating from the equilibrium bidding strategy. $\beta^{M S}$ is bijective, meaning that every sensible bid $b$ can be expressed such that $b=\beta^{M S}(z)$. The bidding function $\beta^{M S}$ constitutes a UPE if and only if the utility function $u_{i}^{M S}\left(v_{i}, b \mid \beta^{M S}\left(v_{i}\right)\right)$ attains its maximum at

\footnotetext{
${ }^{16}$ We consider payment rules based on standard auctions as defined by Krishna (2009). A standard auction is an auction where the highest bidder wins.
} 
$b=\beta^{M S}\left(v_{i}\right)$ for all $v_{i}$. Bidder $i$ 's expected payoff is given by

$$
\begin{aligned}
u^{M S}\left(v_{i}, \beta^{M S}(z) \mid \beta^{M S}\left(v_{i}\right)\right)= & G\left(\beta^{M S}(z)\right) v_{i}-m^{M S}(z) \\
& +\Theta\left(\beta^{M S}(z) \mid \beta^{M S}\left(v_{i}\right)\right) .
\end{aligned}
$$

The first-order condition is given by

$$
\begin{aligned}
\frac{\partial u^{M S}\left(v_{i}, \beta^{M S}(z) \mid \beta^{M S}\left(v_{i}\right)\right)}{\partial z}= & f_{1}^{(N-1)}(z) v_{i}-\frac{\partial}{\partial z} m^{M S}(z) \\
& +\frac{\partial}{\partial z} \Theta\left(\beta^{M S}(z) \mid \beta^{M S}\left(v_{i}\right)\right) \stackrel{!}{=} 0 .
\end{aligned}
$$

In equilibrium it is optimal to report $z=v_{i}$ and it holds that $b^{*}=\beta^{M S}\left(v_{i}\right)$, so we obtain that for all $y$,

$$
\frac{\partial}{\partial y} m^{M S}(z)=f_{1}^{(N-1)}(y) y+\left.\left(\frac{\partial}{\partial y} \Theta\left(\beta^{M S}(y) \mid \beta^{M S}(z)\right)\right)\right|_{z=y} .
$$

This means that

$$
\begin{aligned}
m^{M S}\left(v_{i}\right)= & \underline{m^{M S}}(0)+\int_{0}^{v_{i}} f_{1}^{(N-1)}(y) y d y \\
& +\left.\int_{0}^{v_{i}}\left(\frac{\partial}{\partial y} \Theta\left(\beta^{M S}(y) \mid \beta^{M S}(z)\right)\right)\right|_{z=y} d y .
\end{aligned}
$$

While the right-hand side depends on the multi-stage structure $\mathfrak{M}$, it does not depend on the payment rule $\boldsymbol{\mu}$.

The result holds for $k \geq 1$ stages, so one-stage mechanisms are included. A first application of the RET for loss averse bidders is to rank the English auction with loss averse bidders.

Proposition 3. All standard auction formats yield higher expected revenues with loss averse bidders than the English auction.

From von Wangenheim (2019) we know that the English auction performs worse than the second-price auction revenue-wise. We can apply Proposition 2 to complete the proof. 


\subsection{A robust improvement over one-stage mechanisms}

A mechanism that is to be implemented in real-life and that exploits bidders' loss aversion should not depend on the parameters for loss aversion. An auctioneer cannot hope to be able to accurately estimate these parameters in a way that would help her design a mechanism. We will show that for a parameter space that includes the empirically found loss aversion parameters, it is beneficial for the seller to implement a simple two-stage mechanism for every value realization of every distribution function if there are more than two bidders 17

For an even number of bidders, $2 N$, consider randomly pairing two groups of $N$ bidders and then advance the highest bidder of each pairing to the final. For an odd number of bidders, $2 N+1$, consider randomly pairing of one group of $N$ bidders and one group of $N+1$ bidders. Bidders do not know in which group they are selected, they only know the a priori probability of being in the group with $N$ bidders is 0.5. Again, the highest bidder of each pairing advances to the final. We call this multi-stage structure a tournament, it can be seen in figure 2. We can write $\mathfrak{M}^{T}$ as

$$
\begin{gathered}
\mathfrak{M}^{T, \text { even }}=\{\underbrace{\left\{N,\left\{B_{1}^{(N)}\right\}\right\}}_{\text {Stage } 1}, \underbrace{\left\{2,\left\{B_{1}^{(2)}\right\}\right\}}_{\text {Stage } 2}\} . \\
\mathfrak{M}^{T, \text { odd }}=\{\underbrace{\left\{\left\{N_{P=\frac{1}{2}}, N+1_{P=\frac{1}{2}}\right\},\left\{B_{1}^{\left(N_{P=\frac{1}{2}}, N+1_{P=\frac{1}{2}}\right)}\right\}\right\},\left\{2,\left\{B_{1}^{(2)}\right\}\right\}}_{\text {Stage } 1} \underbrace{\{}_{\text {Stage } 2}\} .
\end{gathered}
$$

As shown in Proposition 2, the payment rule we choose is not relevant for the revenue. For the proof, we choose the first-price auction payment rule.

Proposition 4. Assume an even number of bidders $2 N \geq 4$ that are loss averse in the good domain. Assume that $\lambda \leq \frac{2 N-1}{N-1}$. Then for all $\eta \geq 0$ the revenue is higher in the tournament than in any one-stage mechanism.

Corollary 1. Assume an even number of bidders $2 N \geq 4$ that are loss averse in the

\footnotetext{
${ }^{17}$ See Gächter, Johnson, and Herrmann $(2007)$ for an empirical study on individual-level loss aversion. They present evidence that $\lambda^{g}$ lies around 2 .
} 


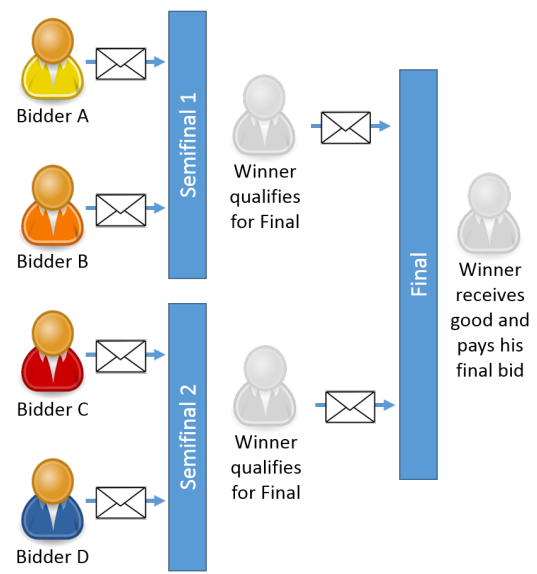

Figure 2: The tournament multi-stage structure $\mathfrak{M}^{T}$ for four bidders.

good domain. Assume that $\lambda \leq \frac{2 N-1}{N-1}$. In the case of the first-price, second-price or all-pay auction as underlying auction format, bids are higher in the tournament than in the corresponding one-stage mechanism for all types.

Proposition 5. Assume an odd number of bidders $2 N+1 \geq 3$ that are loss averse in the good domain. Assume that $\lambda \leq \frac{4 N}{2 N-1}$. Then for all $\eta \geq 0$ the revenue is higher in the tournament than in any one-stage mechanism.

Corollary 2. Assume an even number of bidders $2 N+1 \geq 3$ that are loss averse in the good domain. Assume that $\lambda \leq \frac{4 N}{2 N-1}$. In the case of the first-price, second-price or all-pay auction as underlying auction format, bids are higher in the tournament than in the corresponding one-stage mechanism for all types.

Proposition 6. For $\lambda^{g} \leq 2$, the tournament yields higher bids than the respective one-stage auction for all types.

Consider the first-price auction payment rule. We start with the one-stage mechanism. Assume the other bidders bid according to an increasing, absolutely continuous bidding function $\beta^{F P}$ and let $G(b)=F_{1}^{(N-1)}\left(\beta^{F P^{-1}}(b)\right)$. The expected payment is given by

$$
m^{F P}(b)=G(b) b
$$


The utility function is given by

$$
\begin{aligned}
u_{i}^{F P}\left(v_{i}, b \mid b^{*}\right)= & G(b)(v-b) \\
& +G(b)\left(1-G\left(b^{*}\right)\right) \eta^{g} v \\
& +(1-G(b)) G\left(b^{*}\right) \eta^{g} \lambda^{g}(-v) .
\end{aligned}
$$

The bidding function $\beta^{F P}$ constitutes a UPE if and only if the utility function $u_{i}^{F P}\left(v_{i}, b \mid \beta^{F P}\left(v_{i}\right)\right)$ attains its maximum at $b=\beta^{F P}\left(v_{i}\right)$ for all $v_{i}$. Differentiating $u^{F P}$ with respect to $b$ and plugging in the equilibrium condition $b=\beta^{F P}\left(v_{i}\right)$ yields the ODE,

$$
\beta^{F P^{\prime}}\left(v_{i}\right) F_{1}\left(v_{i}\right)+\beta^{F P}\left(v_{i}\right) f_{1}\left(v_{i}\right) \stackrel{!}{=} v_{i} f_{1}\left(v_{i}\right)\left(1+\left(1-F_{1}\left(v_{i}\right)\right) \eta^{g}+F_{1} \eta^{g} \lambda^{g}\right)
$$

This ODE admits a closed form solution,

$$
\begin{aligned}
\beta^{F P}\left(v_{i}\right) & =\frac{1}{F_{1}\left(v_{i}\right)} \int_{0}^{v_{i}} s f_{1}(s)\left(1+\eta^{g}+F_{1}(s) \eta^{g}\left(\lambda^{g}-1\right)\right) d s \\
& =\frac{1}{F_{1}\left(v_{i}\right)} \int_{0}^{v_{i}} s f_{1}(s)\left(1+\eta^{g}\left(1-F_{1}(s)\right)+\eta^{g} \lambda^{g} F_{1}(s)\right) d s
\end{aligned}
$$

The equilibrium bidding function for the tournament can be derived explicitly, too. With Proposition 1. we can assume bidders bid the same in every stage. Assume that the other bidders bid according to an increasing, absolutely continuous bidding function $\beta^{T}$. In the first stage, bidder $i$ advances if he beats his $N-1$ opponents. This yields

$$
\phi_{1} \circ F=F_{1}^{(N-1)} .
$$

This implies that advancing to the second stage is not informative in any way about the value of the remaining opponent. The intuition behind this can be understood by considering the mechanism with four bidders. Given the bidder won the first round, he may have beaten his toughest opponent already. But he also might have 
beaten the second or third highest bidding one,

Prob(get to 2nd round with $b$ )

$$
\begin{aligned}
& =\frac{1}{3} F_{1}\left(\beta^{T^{-1}}(b)\right)+\frac{2}{3}\left(\frac{1}{2} F_{2}\left(\beta^{T^{-1}}(b)\right)+\frac{1}{2} F_{3}\left(\beta^{T^{-1}}(b)\right)\right) \\
& =F\left(\beta^{T^{-1}}(b)\right) .
\end{aligned}
$$

Given that the bidder reached stage two, the bidder wins if he beats the winner of the second group given he got there,

$$
\begin{aligned}
\phi_{2} \circ F \circ \beta^{T^{-1}}(b) & =\operatorname{Prob}\left(b>\beta^{T}\left(v_{1}^{(N)}\right) \mid b>\beta^{T}\left(v_{1}^{(N-1)}\right)\right) \\
& =\frac{F_{1}^{(N-1)}\left(\beta^{T^{-1}}(b)\right) F_{1}^{(N)}\left(\beta^{T^{-1}}(b)\right)}{F_{1}^{(N-1)}\left(\beta^{T^{-1}}(b)\right)} \\
& =F_{1}^{(N)}\left(\beta^{T^{-1}}(b)\right) .
\end{aligned}
$$

As mentioned before, we have $m^{T}(b)=F_{1}^{(2 N)}\left(\beta^{T^{-1}}(b)\right) b$. Then the utility is given by

$$
\begin{aligned}
& u^{T}\left(v_{i}, b \mid b^{*}\right)=F_{1}^{(2 N-1)}\left(\beta^{T^{-1}}(b)\right)(v-b) \\
& +F_{1}^{(2 N-1)}\left(\beta^{T^{-1}}(b)\right)\left(1-F_{1}^{(N-1)}\left(\beta^{T^{-1}}\left(b^{*}\right)\right)\right) \eta^{g} v_{i} \\
& +F_{1}^{(2 N-1)}\left(\beta^{T^{-1}}(b)\right)\left(1-F_{1}^{(N)}\left(\beta^{T^{-1}}\left(b^{*}\right)\right)\right) \eta^{g} v_{i} \\
& +\left(1-F_{1}^{(N-1)}\left(\beta^{T^{-1}}(b)\right)\right) F_{1}^{(2 N-1)}\left(\beta^{T^{-1}}\left(b^{*}\right)\right)\left(-\eta^{g} \lambda^{g} v_{i}\right) \\
& +\left(F_{1}^{(N-1)}\left(\beta^{T^{-1}}(b)\right)-F_{1}^{(2 N-1)}\left(\beta^{T^{-1}}(b)\right)\right) F_{1}^{(N)}\left(\beta^{T^{-1}}\left(b^{*}\right)\right)\left(-\eta^{g} \lambda^{g} v_{i}\right) .
\end{aligned}
$$

We are interested in finding the equilibrium bidding function for this multi-stage auction. Our equilibrium concept is UPE, this implies that the first-order condition is given by

$$
\left.\left(\frac{\partial u^{T}\left(v_{i}, b \mid b^{*}\right)}{\partial b}\right)\right|_{b^{*}=\beta^{T}\left(v_{i}\right)} \stackrel{!}{=} 0 .
$$


We have

$$
\begin{aligned}
\frac{\partial}{\partial b} u^{T}\left(v_{i}, b \mid b^{*}\right) & \left.\right|_{b^{*}=\beta^{T}\left(v_{i}\right)}=f_{1}^{(2 N-1)}\left(\beta^{T^{-1}}(b)\right)(v-b) \frac{1}{\beta^{T^{\prime}}\left(\beta^{T^{-1}}(b)\right)} \\
& -F_{1}^{(2 N-1)}\left(\beta^{T^{-1}}(b)\right) \\
& +f_{1}^{(2 N-1)}\left(\beta^{T^{-1}}(b)\right)\left(1-F_{1}^{(N-1)}\left(v_{i}\right)\right) \eta^{g} v_{i} \frac{1}{\beta^{T^{\prime}}\left(\beta^{T^{-1}}(b)\right)} \\
& +f_{1}^{(2 N-1)}\left(\beta^{T^{-1}}(b)\right)\left(1-F_{1}^{(N)}\left(v_{i}\right)\right) \eta^{g} v_{i} \frac{1}{\beta^{T^{\prime}}\left(\beta^{T^{-1}}(b)\right)} \\
& +f_{1}^{(N-1)}\left(\beta^{T^{-1}}(b)\right) F_{1}^{(2 N-1)}\left(v_{i}\right) \eta^{g} \lambda^{g} v_{i} \frac{1}{\beta^{T^{\prime}}\left(\beta^{T^{-1}}(b)\right)} \\
& -f_{1}^{(N-1)}\left(\beta^{T^{-1}}(b)\right) F_{1}^{(N)}\left(v_{i}\right) \eta^{g} \lambda^{g} v_{i} \frac{1}{\beta^{T^{\prime}}\left(\beta^{T^{-1}}(b)\right)} \\
& +f_{1}^{(2 N-1)}\left(\beta^{T^{-1}}(b)\right) F_{1}^{(N)}\left(v_{i}\right) \eta^{g} \lambda^{g} v_{i} \frac{1}{\beta^{T^{\prime}}\left(\beta^{T^{-1}}(b)\right)} .
\end{aligned}
$$

In equilibrium it holds that $b=\beta^{T}\left(v_{i}\right)$. The resulting ordinary differential equation for $\beta^{T}$ admits a closed-form solution,

$$
\begin{aligned}
\beta^{T}\left(v_{i}\right)=\frac{1}{F_{1}^{(2 N-1)}\left(v_{i}\right)} \int_{0}^{v_{i}} s & {\left[f_{1}^{(2 N-1)}(s)\right.} \\
& +\eta^{g} f_{1}^{(2 N-1)}(s)\left(2-F_{1}^{(N-1)}(s)-F_{1}^{(N)}(s)\right) \\
+ & \eta^{g} \lambda^{g}\left(f_{1}^{(N-1)}(s) F_{1}^{(2 N-1)}(s)\right. \\
& -f_{1}^{(N-1)}(s) F_{1}^{(N)}(s) \\
& \left.\left.+f_{1}^{(2 N-1)}(s) F_{1}^{(N)}(s)\right)\right] d s .
\end{aligned}
$$

For $\beta^{T}\left(v_{i}\right) \geq \beta^{F P}\left(v_{i}\right)$ to hold for all $v_{i}$, a sufficient condition is that we can rank the arguments of the integrals. As a reminder, $\beta^{F P}\left(v_{i}\right)$ is given by

$$
\beta^{F P}\left(v_{i}\right)=\frac{1}{F_{1}\left(v_{i}\right)} \int_{0}^{v_{i}} s f_{1}(s)\left(1+\eta^{g}\left(1-F_{1}(s)\right)+\eta^{g} \lambda^{g} F_{1}(s)\right) d s
$$

with $F_{1}=F_{1}^{(2 N-1)}$ and $f_{1}=f_{1}^{(2 N-1)}$. Note that the first term stemming from the standard preferences equilibrium bidding function is identical in both bidding functions. What is left are the gain-loss utility terms. This means it has to hold 
that

$$
\begin{array}{r}
\eta^{g} f_{1}^{(2 N-1)}(s)\left(2-F_{1}^{(N-1)}(s)-F_{1}^{(N)}(s)\right) \\
-\eta^{g} f_{1}^{(2 N-1)}(s)\left(1-F_{1}(s)\right) \\
+\eta^{g} \lambda^{g}\left(f_{1}^{(N-1)}(s) F_{1}^{(2 N-1)}(s)-f_{1}^{(N-1)}(s) F_{1}^{(N)}(s)\right. \\
\left.+f_{1}^{(2 N-1)}(s) F_{1}^{(N)}(s)\right) \\
-\eta^{g} \lambda^{g} \eta^{g} f_{1}^{(2 N-1)}(s) F_{1}(s) \stackrel{!}{\geq} 0 .
\end{array}
$$

Note that all terms, except the third one, include $f_{1}^{(2 N-1)}(s)=(2 N-1) F^{2 N-2}(s) f(s)$. Using the definition of the first-order statistic density for distribution functions, we have

$$
\begin{aligned}
f_{1}^{(N-1)}(s) F_{1}^{(2 N-1)}(s) & =(N-1) F^{N-2}(s) f(s) F^{2 N-1}(s) \\
& =\frac{N-1}{2 N-1}(2 N-1) F^{2 N-2}(s) f(s) F^{N-1} \\
& =\frac{N-1}{2 N-1} f_{1}^{(2 N-1)}(s) F_{1}^{(N-1)} .
\end{aligned}
$$

Similarly, we can write the third term as

$$
\begin{aligned}
& f_{1}^{(N-1)}(s) F_{1}^{(2 N-1)}(s)-f_{1}^{(N-1)}(s) F_{1}^{(N)}(s)+f_{1}^{(2 N-1)}(s) F_{1}^{(N)}(s) \\
= & f_{1}^{(2 N-1)}(s)\left(\frac{N-1}{2 N-1} F_{1}^{(N-1)}(s)-\frac{N-1}{2 N-1}+F_{1}^{(N)}(s)\right) .
\end{aligned}
$$

With this, 717 simplifies to

$$
\begin{array}{r}
\left(\lambda^{g}-1\right) F_{1}^{(N)}(s)\left(1-F_{1}^{(N-1)}(s)\right)-\left(\frac{N-1}{2 N-1} \lambda^{g}-1\right)\left(1-F_{1}^{(N-1)}(s)\right) \stackrel{!}{\geq} 0 \\
\Leftrightarrow\left(\lambda^{g}-1\right) F_{1}^{(N)}(s)-\frac{N-1}{2 N-1} \lambda^{g}+1 \stackrel{!}{\geq} 0 \\
\Rightarrow \frac{N-1}{2 N-1} \lambda^{g}-1 \stackrel{!}{\leq} 0 \\
\Leftrightarrow \lambda^{g} \stackrel{!}{\leq} \frac{2 N-1}{N-1}
\end{array}
$$


To prove the corollary, we define

$$
\gamma^{O S}(s)=s\left(1+\eta^{g}\left(1-F_{1}(s)\right)+\eta^{g} \lambda^{g} F_{1}(s)\right) .
$$

Note that $\gamma^{O S}$ is given by the argument of the integral of $\beta^{F P}$. Similarly, define $\gamma^{T}$ as the argument of the integral of $\beta^{T}$. Note that we have shown under which conditions it holds that $\gamma^{O S}(s) \leq \gamma^{T}(s)$. It is straightforward to compute that in the case of the second-price auction payment rule, bidding functions are given by

$$
\begin{gathered}
\beta^{S P}(v)=\gamma^{O S}(v) \\
\beta^{T}(v)=\gamma^{T}(v) .
\end{gathered}
$$

In the case of the all-pay auction, the bidding functions are given by

$$
\begin{aligned}
\beta^{S P}(v) & =\int_{0}^{v} \gamma^{O S}(s) f_{1}(s) d s \\
\beta^{T}(v) & =\int_{0}^{v} \gamma^{T}(s) f_{1}(s) d s .
\end{aligned}
$$

Combining the results from this section concludes the proof to the corollary.

Again, we consider the first-price auction payment rule. We derive the equilibrium bidding function for the tournament with an odd number of bidders in a similar way as for an even number of bidders.

With proposition 1, we can assume bidders bid the same in every stage. Assume the other bidders bid according to an increasing, absolutely continuous bidding function $\beta^{T}$. In the group with $N$ bidders, a bidder advances if he beats his $N-1$ paired opponents. This yields

$$
\phi_{1} \circ F=F^{N-1} .
$$

Given that the bidder reached stage two, the bidder wins if he beats the winner of the second group with $N+1$ bidders, given he got there,

$$
\phi_{2} \circ F \circ \beta^{T^{-1}}(b)=F_{1}^{(N+1)}\left(\beta^{T^{-1}}(b)\right) .
$$


In the group with $N+1$ bidders, a bidder advances if he beats his $N$ paired opponents. This yields

$$
\phi_{1} \circ F=F^{N} .
$$

Given that the bidder reached stage two, the bidder wins if he beats the winner of the second group with $N$ bidders, given he got there,

$$
\phi_{2} \circ F \circ \beta^{T^{-1}}(b)=F_{1}^{(N)}\left(\beta^{T^{-1}}(b)\right) .
$$

Again we have $m^{T}(b)=F_{1}^{(2 N)}\left(\beta^{T^{-1}}(b)\right) b$. Then the utility is given by

$$
\begin{aligned}
u^{T}\left(v_{i}, b \mid\right. & \left.b^{*}\right)=F_{1}^{(2 N)}\left(\beta^{T^{-1}}(b)\right)(v-b) \\
+\frac{1}{2}[ & F_{1}^{(2 N)}\left(\beta^{T^{-1}}(b)\right)\left(1-F_{1}^{(N-1)}\left(\beta^{T^{-1}}\left(b^{*}\right)\right)\right) \eta^{g} v_{i} \\
& +F_{1}^{(2 N)}\left(\beta^{T^{-1}}(b)\right)\left(1-F_{1}^{(N+1)}\left(\beta^{T^{-1}}\left(b^{*}\right)\right)\right) \eta^{g} v_{i} \\
& +\left(1-F_{1}^{(N-1)}\left(\beta^{T^{-1}}(b)\right)\right) F_{1}^{(2 N)}\left(\beta^{T^{-1}}\left(b^{*}\right)\right)\left(-\eta^{g} \lambda^{g} v_{i}\right) \\
& +F_{1}^{(N-1)}\left(\beta^{T^{-1}}(b)\right) F_{1}^{(N+1)}\left(\beta^{T^{-1}}\left(b^{*}\right)\right)\left(-\eta^{g} \lambda^{g} v_{i}\right) \\
& \left.\quad-F_{1}^{(2 N)}\left(\beta^{T^{-1}}(b)\right) F_{1}^{(N+1)}\left(\beta^{T^{-1}}\left(b^{*}\right)\right)\left(-\eta^{g} \lambda^{g} v_{i}\right)\right] \\
+\frac{1}{2}[ & F_{1}^{(2 N)}\left(\beta^{T^{-1}}(b)\right)\left(1-F_{1}^{(N)}\left(\beta^{T^{-1}}\left(b^{*}\right)\right)\right) \eta^{g} v_{i} \\
& +F_{1}^{(2 N)}\left(\beta^{T^{-1}}(b)\right)\left(1-F_{1}^{(N)}\left(\beta^{T^{-1}}\left(b^{*}\right)\right)\right) \eta^{g} v_{i} \\
& +\left(1-F_{1}^{(N)}\left(\beta^{T^{-1}}(b)\right)\right) F_{1}^{(2 N)}\left(\beta^{T^{-1}}\left(b^{*}\right)\right)\left(-\eta^{g} \lambda^{g} v_{i}\right) \\
& +F_{1}^{(N)}\left(\beta^{T^{-1}}(b)\right) F_{1}^{(N)}\left(\beta^{T^{-1}}\left(b^{*}\right)\right)\left(-\eta^{g} \lambda^{g} v_{i}\right) \\
& \left.-F_{1}^{(2 N)}\left(\beta^{T^{-1}}(b)\right) F_{1}^{(N)}\left(\beta^{T^{-1}}\left(b^{*}\right)\right)\left(-\eta^{g} \lambda^{g} v_{i}\right)\right]
\end{aligned}
$$

The bracketed expression starting in the second line accounts for the case the bidder is sorted into the $N$-bidder group, the bracketed expression starting in the seventh line accounts for the case the bidder is sorted into the $N+1$-bidder group. We are interested in finding the equilibrium bidding function for this multi-stage auction. Our equilibrium concept is UPE, this implies that the first-order condition is given 
by

$$
\left.\left(\frac{\partial u^{T}\left(v_{i}, b \mid b^{*}\right)}{\partial b}\right)\right|_{b^{*}=\beta^{T}\left(v_{i}\right)} \stackrel{!}{=} 0 .
$$

Leaving out the arguments of the functions for the sake of readability, we have

$$
\begin{aligned}
& \left.\beta^{T^{\prime}}\left(\beta^{T^{-1}}\right) \cdot \frac{\partial}{\partial b} u^{T}\left(v_{i}, b \mid b^{*}\right)\right|_{b^{*}=\beta^{T}\left(v_{i}\right)}=f_{1}^{(2 N)}(v-b)-F_{1}^{(2 N-1)} \beta^{T^{\prime}}\left(\beta^{T^{-1}}\right) \\
& +\frac{1}{2}\left[f_{1}^{(2 N)}\left(1-F_{1}^{(N-1)}\right) \eta^{g} v_{i}+f_{1}^{(2 N)}\left(1-F_{1}^{(N+1)}\right) \eta^{g} v_{i}\right. \\
& \left.+f_{1}^{(N-1)} F_{1}^{(2 N)} \eta^{g} \lambda^{g} v_{i}-\left(f_{1}^{(N-1)}-f_{1}^{(2 N)}\right) F_{1}^{(N+1)} \eta^{g} \lambda^{g} v_{i}\right] \\
& +\frac{1}{2}\left[f_{1}^{(2 N)}\left(1-F_{1}^{(N)}\right) \eta^{g} v_{i}+f_{1}^{(2 N)}\left(1-F_{1}^{(N)}\right) \eta^{g} v_{i}\right. \\
& \left.+f_{1}^{(N)} F_{1}^{(2 N)} \eta^{g} \lambda^{g} v_{i}-\left(f_{1}^{(N)}-f_{1}^{(2 N)}\right) F_{1}^{(N)} \eta^{g} \lambda^{g} v_{i}\right] .
\end{aligned}
$$

In equilibrium it holds that $b=\beta^{T}\left(v_{i}\right)$. The resulting ordinary differential equation for $\beta^{T}$ admits a closed form solution,

$$
\begin{aligned}
\beta^{T}\left(v_{i}\right)= & \frac{1}{F_{1}^{(2 N)}\left(v_{i}\right)} \int_{0}^{v_{i}} s\left[f_{1}^{(2 N)}(s)\right. \\
& +\frac{\eta^{g}}{2} f_{1}^{(2 N)}(s)\left(4-F_{1}^{(N-1)}(s)-F_{1}^{(N+1)}(s)-2 F_{1}^{(N)}(s)\right) \\
& +\frac{\eta^{g} \lambda^{g}}{2}\left(f_{1}^{(N-1)}(s) F_{1}^{(2 N)}(s)+f_{1}^{(N)}(s) F_{1}^{(2 N)}(s)\right. \\
& \quad-\left(f_{1}^{(N-1)}(s)-f_{1}^{(2 N)}(s)\right) F_{1}^{(N+1)}(s) \\
& \left.\left.\quad-\left(f_{1}^{(N)}(s)-f_{1}^{(2 N)}(s)\right) F_{1}^{(N)}(s)\right)\right] d s .
\end{aligned}
$$

Again, as a sufficient condition we want to show that we can rank the arguments of the integrals. The equilibrium bidding function of the first-price auction is now given by

$$
\beta^{F P}\left(v_{i}\right)=\frac{1}{F_{1}^{(2 N)}\left(v_{i}\right)} \int_{0}^{v_{i}} s f_{1}^{(2 N)}(s)\left(1+\eta^{g}\left(1-F_{1}^{(2 N)}(s)\right)+\eta^{g} \lambda^{g} F_{1}^{(2 N)}(s)\right) d s . \quad(94)
$$

As before, the first term stemming from the standard preferences equilibrium bid- 
ding function is identical in both bidding functions. What is left are the gain-loss utility terms. This means it has to hold that,

$$
\begin{array}{r}
\frac{\eta^{g}}{2} f_{1}^{(2 N)}(s)\left(4-F_{1}^{(N-1)}(s)-F_{1}^{(N+1)}(s)-2 F_{1}^{(N)}(s)\right) \\
-\eta^{g} f_{1}^{(2 N)}(s)\left(1-F_{1}^{(2 N)}(s)\right) \\
+\frac{\eta^{g} \lambda^{g}}{2}\left[f_{1}^{(N-1)}(s) F_{1}^{(2 N)}(s)+f_{1}^{(N)}(s) F_{1}^{(2 N)}(s)\right. \\
-\left(f_{1}^{(N-1)}(s)-f_{1}^{(2 N)}(s)\right) F_{1}^{(N+1)}(s) \\
\left.-\left(f_{1}^{(N)}(s)-f_{1}^{(2 N)}(s)\right) F_{1}^{(N)}(s)\right] \\
-\eta^{g} \lambda^{g} \eta^{g} f_{1}^{(2 N)}(s) F_{1}^{(2 N)}(s) \stackrel{!}{\geq} 0 .
\end{array}
$$

Note that all terms, except the third one, include $f_{1}^{(2 N)}(s)=2 N F^{2 N}(s) f(s)$. Using the definition of the first-order statistic density for distribution functions and leaving the arguments of the functions out, we can write the third term as

$$
\begin{aligned}
& f_{1}^{(N-1)} F_{1}^{(2 N)}+f_{1}^{(N)} F_{1}^{(2 N)}-\left(f_{1}^{(N-1)}-f_{1}^{(2 N)}\right) F_{1}^{(N+1)} \\
&-\left(f_{1}^{(N)}-f_{1}^{(2 N)}\right) F_{1}^{(N)} \\
&=f_{1}^{(2 N)}\left(\frac{N-1}{2 N} F_{1}^{(N-1)}+\frac{1}{2} F_{1}^{(N)}-\frac{N-1}{2 N}+F_{1}^{(N+1)}-\frac{1}{2}+F_{1}^{(N)}\right) .
\end{aligned}
$$

This inequality can be solved analytically for three bidders and has to be solved numerically for more than three bidders. For three bidders the inequality simplifies to

$$
\frac{1}{2}-F+\frac{1}{2} F^{2}+\lambda^{g}\left(-\frac{1}{4}+\frac{3}{4} F-\frac{1}{2} F^{2}\right) \stackrel{!}{>} 0 .
$$

Since only $F$ appears, but not its argument, we can solve the inequality without inverting $F$. The extremum of the left-hand side is attained at $F=\frac{3 \lambda-4}{4(\lambda-1)}$, but since the coefficient of the $F^{2}$-terms is given by $\frac{1}{2}(1-\lambda)$, this is a maximum. This means that the minimum for valid valued of $F$ is at $F=0$ or $F=1$. For $F=1$, 


\begin{tabular}{c|c|c|c|c|c|c}
$N$ & 1 & 2 & 3 & 4 & 5 & 6 \\
\hline Total bidders & 3 & 5 & 7 & 9 & 11 & 13 \\
\hline$\lambda^{\text {crit }}$ & 2.0 & 2.6484 & 2.3995 & 2.2856 & 2.2222 & 2.1818 \\
\hline$\frac{4 N}{2 N-1}$ & 4.0 & 2.6667 & 2.4000 & 2.2857 & 2.2222 & 2.1818 \\
\hline
\end{tabular}

Table 1: Critical $\lambda^{g}$ values for different number of bidders.

the left-hand side is always equal to zero. For $F=0$, we have

$$
\frac{2-\lambda}{4} \stackrel{!}{>} 0 .
$$

This is fulfilled for all $\lambda \leq 2$. For $N>1$, meaning $5,7,9 \ldots$ bidders, an analytic solution is not tractable. The inequality can however be solved numerically. The results can be found in Table 1 , the code to compute the critical lambdas can be found in Appendix 6.1.

From the proof of the case with an even number of bidders, one might expect that the critical $\lambda^{g}$-values are given by the expression for an even number of bidders plus half a bidder per group in expectation,

$$
\frac{2\left(N+\frac{1}{2}\right)-1}{N+\frac{1}{2}-1}=\frac{4 N}{2 N-1} .
$$

While this expression closely approximates the critical $\lambda^{g}$ S for more than four bidders, the actual $\lambda^{g}$-values are somewhat smaller than this, as can be seen in Table 1 This is due to the fact that the order statistics for the $N+1$ - and $N$-bidder groups depend non-linearly on the number of bidders.

The corollary is proven the exact same way as in the case for an even number of bidders.

The minimal critical $\lambda^{g}$ is given by $\lambda^{g}=2$. Together with Proposition 4 and Proposition 5, this means that for $N \geq 3$ bidders, an auctioneer is always better off if she conducts a tournament instead of the corresponding one-stage mechanism.

Note that we derived the critical $\lambda^{g}$-values such that every type bids higher in 
the tournament than in the corresponding one-stage mechanism. If the auctioneer is solely interested in expected revenue, then the critical $\lambda^{g}$-values are significantly higher but depend on the distribution function and generally need to be determined numerically.

An exception is the case for $N=4$ bidders and the uniform distribution. Here, the difference between the expected payment in tournament vs the corresponding one-stage mechanism is given by

$$
\mathbb{E}\left[m^{T}-m^{F P}\right]=\frac{1}{840} \eta^{g}\left(\lambda^{g}+27\right)
$$

This expression is strictly positive for all admissible $\lambda^{g}$ and $\eta^{g}$, meaning that the tournament always yields higher revenues than the corresponding one-stage mechanism in this setting. The same result can be derived for a total of three bidders in the case of uniformly distributed values. For $N>4$, the critical $\lambda^{g}$-values have to be determined numerically, even for the uniform distribution.

\subsection{Optimal efficient two-stage mechanism}

We have already shown that the tournament poses a strict improvement over onestage mechanisms if the auctioneer is facing loss averse bidders. Restricting ourselves to two stages, one might ask what the optimal efficient mechanism looks like. In this section we derive and discuss the optimal efficient two-stage mechanism.

Proposition 7. Assume bidders are loss averse in the good domain and assume a general two-stage mechanism $(\boldsymbol{\mu}, \mathfrak{M})$ that induces $\varphi_{1}(s)=\phi_{1} \circ F(s)$ and $\varphi_{2}(s)=$ $\phi_{2} \circ F(s)$. Then the expected payment of a bidder with value $v$ is given by

$$
\begin{array}{rl}
m^{T S}(v)=\int_{0}^{v} & s\left(f_{1}(s)+\eta^{g} f_{1}(s)\left[2-\varphi_{1}(s)-\varphi_{2}(s)\right]\right. \\
& \left.+\eta^{g} \lambda^{g}\left[F_{1}(s) \varphi_{1}^{\prime}(s)+f_{1}(s) \varphi_{2}(s)-\varphi_{1}^{\prime}(s) \varphi_{2}(s)\right]\right) d s .
\end{array}
$$

We start the proof by choosing the first-price payment rule. We will then use Proposition 2 to show that we can choose any standard payment rule after we have derived the two-stage structure $\mathfrak{M}$. With proposition 1, we can assume bidders bid the same in every stage. Assume the other bidders bid according to an increasing, 
absolutely continuous bidding function $\beta^{T S}$. Note that

$$
\varphi_{1}\left(\beta^{T S^{-1}}(b)\right) \varphi_{2}\left(\beta^{T S^{-1}}(b)\right)=F_{1}\left(\beta^{T S^{-1}}(b)\right)
$$

Then the utility is given by

$$
\begin{aligned}
& u^{T S}\left(v_{i}, b \mid b^{*}\right)=F_{1}\left(\beta^{T S^{-1}}(b)\right)\left(v_{i}-b\right) \\
& \quad+F_{1}\left(\beta^{T S^{-1}}(b)\right)\left(1-\varphi_{1}\left(\beta^{T S^{-1}}\left(b^{*}\right)\right)\right) \eta^{g} v \\
& \quad+F_{1}\left(\beta^{T S^{-1}}(b)\right)\left(1-\varphi_{2}\left(\beta^{T S^{-1}}\left(b^{*}\right)\right)\right) \eta^{g} v \\
& \quad+\left(1-\varphi_{1}\left(\beta^{T S^{-1}}(b)\right)\right) F_{1}\left(\beta^{T S^{-1}}\left(b^{*}\right)\right) \eta^{g} \lambda(-v) \\
& \quad+\left(\varphi_{1}\left(\beta^{T S^{-1}}(b)\right)-F_{1}\left(\beta^{T S^{-1}}(b)\right)\right) \varphi_{2}\left(\beta^{T S^{-1}}\left(b^{*}\right)\right) \eta^{g} \lambda(-v)
\end{aligned}
$$

The bidding function $\beta^{T S}$ constitutes a UPE if and only if the utility function $u_{i}^{T S}\left(v_{i}, b \mid \beta^{T S}\left(v_{i}\right)\right)$ attains its maximum at $b=\beta^{T S}\left(v_{i}\right)$ for all $v_{i}$. Differentiating $u^{T S}$ with respect to $b$ and plugging in the equilibrium condition $b=\beta^{F P}\left(v_{i}\right)$ yields the ODE

$$
\begin{gathered}
F_{1}(s) \beta^{T S}(s)+f_{1}(s) \beta^{T S^{\prime}}(s)=s\left(f_{1}(s)+\eta^{g} f_{1}(s)\left[2-\varphi_{1}(s)-\varphi_{2}(s)\right]\right. \\
\left.+\eta^{g} \lambda^{g}\left[F_{1}(s) \varphi_{1}^{\prime}(s)+f_{1}(s) \varphi_{2}(s)-\varphi_{1}^{\prime}(s) \varphi_{2}(s)\right]\right)
\end{gathered}
$$

It follows that

$$
\begin{array}{rl}
\beta^{T S}(v)=\frac{1}{F_{1}(v)} \int_{0}^{v} & s\left(f_{1}(s)+\eta^{g} f_{1}(s)\left[2-\varphi_{1}(s)-\varphi_{2}(s)\right]\right. \\
& \left.+\eta^{g} \lambda^{g}\left[F_{1}(s) \varphi_{1}^{\prime}(s)+f_{1}(s) \varphi_{2}(s)-\varphi_{1}^{\prime}(s) \varphi_{2}(s)\right]\right) d s
\end{array}
$$


and

$$
\begin{array}{rl}
m^{T S}(v)=\int_{0}^{v} & s\left(f_{1}(s)+\eta^{g} f_{1}(s)\left[2-\varphi_{1}(s)-\varphi_{2}(s)\right]\right. \\
& \left.+\eta^{g} \lambda^{g}\left[F_{1}(s) \varphi_{1}^{\prime}(s)+f_{1}(s) \varphi_{2}(s)-\varphi_{1}^{\prime}(s) \varphi_{2}(s)\right]\right) d s
\end{array}
$$

Proposition 8. Assume bidders are loss averse in the good domain and assume a general two-stage mechanism that induces $\varphi_{1}(s)=\phi_{1} \circ F(s)$ and $\varphi_{2}(s)=\phi_{2} \circ F(s)$. Then the expected revenue for the auctioneer is given by

$$
\begin{array}{rl}
\mathbb{E}[R]=N \int_{0}^{1} & s(1-F(s))\left(f_{1}(s)+\eta^{g} f_{1}(s)\left[2-\varphi_{1}(s)-\varphi_{2}(s)\right]\right. \\
& \left.+\eta^{g} \lambda^{g}\left[F_{1}(s) \varphi_{1}^{\prime}(s)+f_{1}(s) \varphi_{2}(s)-\varphi_{1}^{\prime}(s) \varphi_{2}(s)\right]\right) d s .
\end{array}
$$

Again, assume the other bidders bid according to an increasing, absolutely continuous bidding function $\beta^{T S}$ and use the interim results of Proposition 7. Define

$$
\begin{aligned}
\Gamma(s)= & s\left(f_{1}(s)+\eta^{g} f_{1}(s)\left[2-\varphi_{1}(s)-\varphi_{2}(s)\right]\right. \\
& \left.+\eta^{g} \lambda^{g}\left[F_{1}(s) \varphi_{1}^{\prime}(s)+f_{1}(s) \varphi_{2}(s)-\varphi_{1}^{\prime}(s) \varphi_{2}(s)\right]\right) .
\end{aligned}
$$

The expected revenue is given by

$$
\mathbb{E}[R]=N \int_{0}^{1} \int_{0}^{v} \Gamma(s) d s f(v) d v .
$$

Partial integration yields

$$
\begin{aligned}
\int_{0}^{1} \int_{0}^{v} \Gamma(s) d s f(v) d v & =\left[\int_{0}^{v} \Gamma(s) d s F(v)\right]_{v=0}^{v=1}-\int_{0}^{1} \Gamma(s) F(s) d s \\
& =\int_{0}^{1} \Gamma(s) d s-\int_{0}^{1} \Gamma(s) F(s) d s \\
& =\int_{0}^{1}(1-F(s)) \Gamma(s) d s .
\end{aligned}
$$

Proposition 9 (Optimal two-stage structure). Assume bidders are loss averse in the good domain. Then the optimal two-stage structure is given by 
Stage 1: With probability $\frac{1}{\lambda}$ bidders get to the second stage with probability 1. With probability $\frac{\lambda-1}{\lambda}$ only the strongest bidder advances to stage 2 and has thus won the auction.

Stage 2: If bidders got to stage 2 with probability 1, the strongest bidder wins the auction.

Bidders are left unaware whether the branch in which everyone advances to the second stage was selected or if the auction took place in the first stage. The only information they receive is whether they have reached stage two or not and after the second stage, whether they have won the auction or not. The interpretation here is that this mechanism induces just the right amount of risk, a bidder in stage 2 does not know whether he beat his opponents already or if the "real" auction is yet to come. This takes care of lower types who do not need to insure themselves against their expectations by bidding even lower, while it encourages strong bidders to bid even higher. The proof is structured in two parts. In a first step we optimize the expected revenue functional for general distribution functions and $\varphi_{1}$ and $\varphi_{2}$. In the second step, we show that the optimal $\varphi_{i}$-functions are equivalent to admissible $\varphi_{i}$, meaning that they satisfy the conditions from section 3.2 Assume the other bidders bid according to an increasing, absolutely continuous bidding function $\beta^{T S}$ and use the interim results of Proposition 7.

We have

$$
\mathbb{E}[R]=N \int_{0}^{1}(1-F(s)) \Gamma(s) d s=: N \int_{0}^{1} J\left(s, \varphi_{1}, \varphi_{1}^{\prime}, \varphi_{2}\right) d s
$$

with

$$
\begin{aligned}
\Gamma(s)= & s\left(f_{1}(s)+\eta^{g} f_{1}(s)\left[2-\varphi_{1}(s)-\varphi_{2}(s)\right]\right. \\
& \left.+\eta^{g} \lambda^{g}\left[F_{1}(s) \varphi_{1}^{\prime}(s)+f_{1}(s) \varphi_{2}(s)-\varphi_{1}^{\prime}(s) \varphi_{2}(s)\right]\right) .
\end{aligned}
$$

We need to find $\varphi_{1}$ and $\varphi_{2}$ that maximize the functional

$$
\int_{0}^{1} J\left(s, \varphi_{1}, \varphi_{1}^{\prime}, \varphi_{2}\right) d s
$$

A candidate for the optimal $\varphi_{i}$ is given by solving the constrained Euler-Lagrange 
equations for our functional. We will nonetheless begin with the unconstrained Euler-Lagrange equations,

$$
\left\{\begin{aligned}
\frac{\partial}{\partial \varphi_{1}} J\left(s, \varphi_{1}, \varphi_{1}^{\prime}, \varphi_{2}\right)-\frac{d}{d s}\left(\frac{\partial}{\partial \varphi_{1}^{\prime}} J\left(s, \varphi_{1}, \varphi_{1}^{\prime}, \varphi_{2}\right)\right) & =0 \\
\frac{\partial}{\partial \varphi_{2}} J\left(s, \varphi_{1}, \varphi_{1}^{\prime}, \varphi_{2}\right)-\frac{d}{d s}\left(\frac{\partial}{\partial \varphi_{2}^{\prime}} J\left(s, \varphi_{1}, \varphi_{1}^{\prime}, \varphi_{2}\right)\right) & =0 \\
\varphi_{1}(1) & =1 \\
\varphi_{2}(1) & =1 .
\end{aligned}\right.
$$

The initial values of the $\varphi_{i}$ are the only natural choice: For reasons of efficiency, the highest possible type should always advance with certainty. The probability that two bidders are of the highest possible type is zero. Prescribing values for $\varphi_{i}(0)$ could lead to distortions since it might be optimal to have an atom on 0 . Note that $J$ does not depend on $\varphi_{2}^{\prime}$, so the Euler-Lagrange equations simplify to

$$
\left\{\begin{aligned}
\frac{\partial}{\partial \varphi_{1}} J\left(s, \varphi_{1}, \varphi_{1}^{\prime}, \varphi_{2}\right)-\frac{d}{d s}\left(\frac{\partial}{\partial \varphi_{1}^{\prime}} J\left(s, \varphi_{1}, \varphi_{1}^{\prime}, \varphi_{2}\right)\right) & =0 \\
\frac{\partial}{\partial \varphi_{2}} J\left(s, \varphi_{1}, \varphi_{1}^{\prime}, \varphi_{2}\right) & =0 \\
\varphi_{1}(1) & =1 \\
\varphi_{2}(1) & =1 .\left(a_{1}\right)
\end{aligned}\right.
$$

This system of ordinary differential equations is closed-form solvable for general 
distribution functions. We begin with the initial value problem $\left(b_{1}\right),\left(a_{2}\right),\left(b_{2}\right)$.

$$
\begin{aligned}
& \left\{\begin{aligned}
s(1-F(s))\left[-\eta^{g} f_{1}(s)+\eta^{g} \lambda^{g}\left(f_{1}(s)-\varphi_{1}^{\prime}(s)\right)\right] & =0 & \left(b_{1}\right) \\
\varphi_{1}(1) & =1 & \left(b_{2}\right) \\
\varphi_{2}(1) & =1 & \left(b_{2}\right)
\end{aligned}\right. \\
& \Leftrightarrow \begin{cases}\varphi_{1}^{\prime}(s)=\frac{f_{1}(s)\left(\lambda^{g}-1\right)}{\lambda} & \left(b_{1}\right) \\
\varphi_{1}(1)=1 & \left(b_{2}\right)\end{cases} \\
& \Rightarrow \quad \varphi_{1}(s)=\frac{1+F_{1}(s)\left(\lambda^{g}-1\right)}{\lambda^{g}} .
\end{aligned}
$$

For the second initial value problem $\left(a_{1}\right),\left(a_{2}\right),\left(b_{2}\right)$, we have

$$
\left\{\begin{aligned}
s(1-F(s))\left[-\eta^{g} f_{1}(s)-\eta^{g} \lambda^{g}\left(f_{1}(s)-\varphi_{2}^{\prime}(s)\right)\right] & \\
-\eta^{g} \lambda^{g}(1-F(s)-s f(s))\left(F_{1}(s)-\varphi_{2}(s)\right) & =0 \\
\varphi_{1}(1) & =1 . \\
\varphi_{2}(1) & =1 .
\end{aligned}\right.
$$

Note that the ODE only depends on $\varphi_{2}$, as was the case with $\left(b_{1}\right),\left(a_{2}\right),\left(b_{2}\right)$ and $\varphi_{1}$. After rearranging and applying the product rule, we arrive at

$$
\varphi_{2}(s)=F_{1}(s)-\frac{1}{s(1-F(s))} \int_{s}^{1} \frac{y(1-F(y)) f_{1}(y)}{\lambda^{g}} d y .
$$

This means that for the unconstrained optimization problem, the solution is given by

$$
\left\{\begin{array}{l}
\varphi_{1}(s)=\frac{1+F_{1}(s)\left(\lambda^{g}-1\right)}{\lambda^{g}} \\
\varphi_{2}(s)=F_{1}(s)-\frac{1}{s(1-F(s))} \int_{s}^{1} \frac{y(1-F(y)) f_{1}(y)}{\lambda^{g}} d y .
\end{array}\right.
$$

Note that $\varphi_{1}(s) \varphi_{s}(s) \neq F_{1}(s)$, meaning that these do not satisfy the conditions from section 3.2 . We now show that choosing $\varphi_{1}(s)$ and $\varphi_{2}(s)$ according to the solutions of the unconstrained Euler-Lagrange equations is equivalent to choosing 
$\varphi_{2}(s)=\frac{F_{1}(s)}{\varphi_{1}(s)}$.

Choosing $\varphi_{1}(s)$ according to 123 , the expressions of $\int_{0}^{1} J\left(s, \varphi_{1}, \varphi_{1}^{\prime}, \varphi_{2}\right) d s$ that involve $\varphi_{2}(s)$ are given by

$$
\begin{aligned}
& \int_{0}^{1} s(1-F(s)) \eta^{g} \varphi_{2}(s)\left[-f_{1}(s)+\lambda^{g} f_{1}(s)-\lambda^{g} \varphi_{1}^{\prime}(s)\right] d s \\
= & \int_{0}^{1} s(1-F(s)) \eta^{g} \varphi_{2}(s)\left[f_{1}(s)\left(\lambda^{g}-1\right)-\not \chi^{g} \frac{f_{1}(s)\left(\lambda^{g}-1\right)}{\chi^{g}}\right] d s \\
= & 0 .
\end{aligned}
$$

This implies that once we have chosen $\varphi_{1}(s)$ as the solution of the unconstrained optimization problem and therefore independent of $\varphi_{2}(s)$, it does not matter which $\varphi_{2}(s)$ we choose, as long as it remains measurable. Therefore our final $\varphi_{i}$ are given by

$$
\left\{\begin{array}{l}
\varphi_{1}(s)=\frac{1+F_{1}(s)\left(\lambda^{g}-1\right)}{\lambda^{g}} \\
\varphi_{2}(s)=\frac{\lambda^{g} F_{1}(s)}{1+F_{1}(s)\left(\lambda^{g}-1\right)} .
\end{array}\right.
$$

This two-stage structure optimizes the revenue for the seller. We can even show that bids of all types are higher than in the one-stage variants of the mechanism and not just overall revenue.

Proposition 10. Assume bidders are loss averse in the good domain and consider either the first-price auction, the second-price auction or the all-pay auction. Equilibrium bids in the optimal two-stage structure are higher than in the corresponding one-stage mechanism.

First note that replacing the $\varphi_{i}$ in $\Gamma$ by 127 yields

$$
\begin{aligned}
\Gamma(s)= & s\left(f_{1}(s)+\eta^{g} f_{1}(s)\left[2-\varphi_{1}(s)-\varphi_{2}(s)\right]\right. \\
& \left.+\eta^{g} \lambda^{g}\left[F_{1}(s) \varphi_{1}^{\prime}(s)+f_{1}(s) \varphi_{2}(s)-\varphi_{1}^{\prime}(s) \varphi_{2}(s)\right]\right) \\
& =s f_{1}(s)\left(1+\eta^{g}\left(2-\frac{1}{\lambda^{g}}\right)+\eta^{g} \frac{\left(\lambda^{g}-1\right)^{2}}{\lambda} F_{1}(s)\right) .
\end{aligned}
$$


Define

$$
\begin{aligned}
& \gamma^{O S}(s)=s\left(1+\eta^{g}\left(1-F_{1}(s)\right)+\eta^{g} \lambda^{g} F_{1}(s)\right) \\
& \gamma^{O p t}(s)=s\left(1+\eta^{g}\left(2-\frac{1}{\lambda^{g}}\right)+\eta^{g} \frac{\left(\lambda^{g}-1\right)^{2}}{\lambda} F_{1}(s)\right) .
\end{aligned}
$$

We have

$$
\begin{gathered}
\gamma^{O p t}(s) \stackrel{!}{\geq} \gamma^{O S}(s) \\
\Leftrightarrow \quad 2-\frac{1}{\lambda^{g}}+\frac{\left(\lambda^{g}-1\right)^{2}}{\lambda} F_{1}(s) \stackrel{!}{\geq} 1-F_{1}(s)+\lambda^{g} F_{1}(s) \\
\Leftrightarrow \quad F_{1}(s)-1 \stackrel{!}{\leq} 0,
\end{gathered}
$$

which is always true. This means that the ranking holds for the first-price auction. One can easily compute that in the case of the second-price auction as underlying mechanism, bidding functions are given by

$$
\begin{aligned}
& \beta^{S P}(v)=\gamma^{O S}(v) \\
& \beta^{O p t}(v)=\gamma^{O p t}(v) .
\end{aligned}
$$

In the case of the all-pay auction, the bidding functions are given by

$$
\begin{aligned}
& \beta^{S P}(v)=\int_{0}^{v} \gamma^{O S}(s) f_{1}(s) d s \\
& \beta^{O p t}(v)=\int_{0}^{v} \gamma^{O p t}(s) f_{1}(s) d s .
\end{aligned}
$$

This concludes the proof. 


\section{Conclusion}

In this paper we investigate how a buyer should design her procurement mechanism when bidders are loss averse. Loss aversion implies that the willingness to pay of a bidder depends on the probability he assigns to winning the auction. We show that a simple two-stage mechanism, the tournament, outperforms any one-stage mechanism revenue-wise if bidders are not too loss averse. As a robustness-check, we show that the buyer's revenue is not dependent on the payment rule she implements. Once the structure of the multi-stage mechanism is fixed, a revenue equivalence principle holds. Finally, we derive the optimal, efficient two-stage mechanism. This mechanism is, in contrast to the tournament, dependent on the degree of loss aversion of the bidders and therefore difficult to implement in real-life procurement.

Our analysis opens the door to further research. On the one hand, it might be interesting to investigate whether a buyer could further improve her revenue if she were to implement a three-stage (or even more stages) mechanism. Numerical simulations suggest that the answer is no, but the problem quickly becomes untractable even for a fixed cost distribution like the uniform distribution. On the other hand, one could expand the model to include bidders that are loss averse in the money domain, too. The revenue equivalence principle that we derived fails in that case, as shown by Eisenhuth and Ewers (2012). In their paper, they show that the all-pay auction yields higher revenues than the first-price auction in a setting similar to ours. This implies that the optimal mechanism for two or more stages will depend on the payment rule the buyer implements, making the optimization problem a lot harder. 


\section{Appendix}

\subsection{Mathematica code}

The code takes a starting value an then shoots $\lambda$-values until the minimum of the function Func crosses 0.

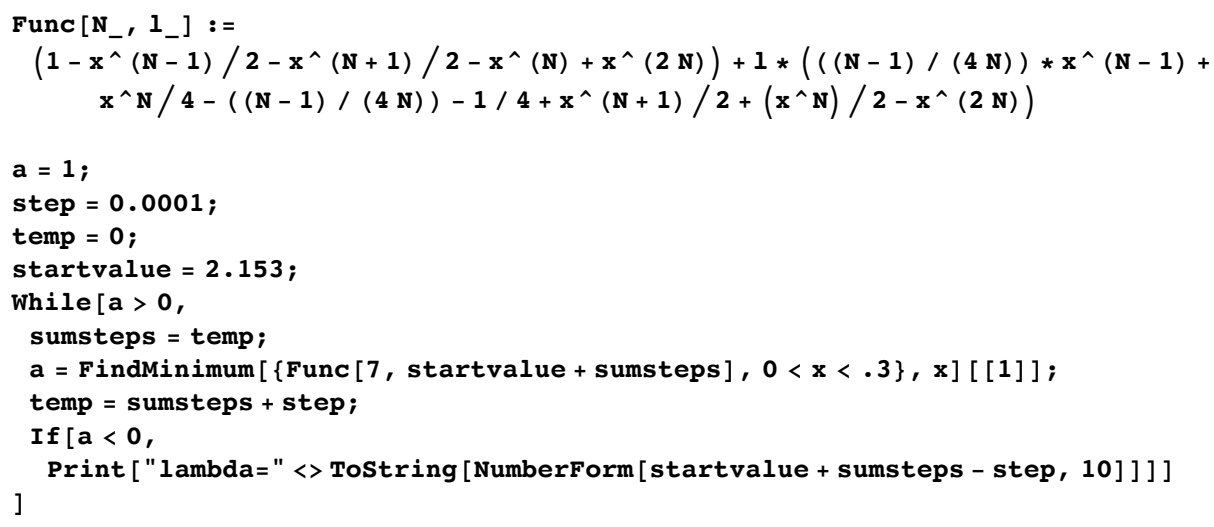




\section{References}

BanerJi, A., And N. Gupta (2014): "Detection, identification, and estimation of loss aversion: Evidence from an auction experiment," American Economic Journal: Microeconomics, 6(1), 91-133.

Ehrhart, K., AND M. Otт (2014): "Reference-dependent bidding in dynamic auctions," Discussion paper, working paper.

EISEnhuth, R., AND M. Ewers (2012): "Auctions with loss averse bidders," Discussion paper, Citeseer.

Engelbrecht-Wiggans, R., AND E. KatoK (2007): "Regret in auctions: theory and evidence," Economic Theory, 33(1), 81-101.

Gächter, S., E. J. Johnson, And A. Herrmann (2007): "Individual-level loss aversion in riskless and risky choices," .

Heffetz, O., And J. A. List (2014): "Is the endowment effect an expectations effect?," Journal of the European Economic Association, 12(5), 1396-1422.

Herweg, F., D. Müller, And P. Weinschenk (2010): "Binary payment schemes: Moral hazard and loss aversion," American Economic Review, 100(5), $2451-77$.

Horowitz, J. K., And K. E. McConnell (2003): "Willingness to accept, willingness to pay and the income effect," Journal of Economic Behavior \& Organization, 51(4), 537-545.

Kahneman, D., J. L. Knetsch, And R. H. Thaler (1990): "Experimental tests of the endowment effect and the Coase theorem," Journal of political Economy, 98(6), 1325-1348.

Kőszegi, B., AND M. RABIN (2006): "A model of reference-dependent preferences," The Quarterly Journal of Economics, 121(4), 1133-1165.

Krishna, V. (2009): Auction theory. Academic press. 
Lange, A., And A. Ratan (2010): "Multi-dimensional reference-dependent preferences in sealed-bid auctions-How (most) laboratory experiments differ from the field," Games and Economic Behavior, 68(2), 634-645.

Maskin, E., AND J. Riley (1984): "Optimal Auctions with Risk Averse Buyers," Econometrica, 52(6), 1473-1518.

Rosato, A., And A. A. Tymula (2019): "Loss aversion and competition in Vickrey auctions: Money ain't no good," Games and Economic Behavior, 115, 188-208.

von Wangenheim, J. (2019): "English versus Vickrey Auctions with Loss Averse Bidders," Discussion paper, CRC TRR 190 Rationality and Competition. 
Download ZEW Discussion Papers from our ftp server:

http://ftp.zew.de/pub/zew-docs/dp/

or see:

https://www.ssrn.com/link/ZEW-Ctr-Euro-Econ-Research.html

https://ideas.repec.org/s/zbw/zewdip.html

$$
\text { // }
$$

IMPRINT

ZEW - Leibniz-Zentrum für Europäische Wirtschaftsforschung GmbH Mannheim

ZEW - Leibniz Centre for European

Economic Research

L 7,1 68161 Mannheim · Germany

Phone +49621 1235-01

info@zew.de·zew.de

Discussion Papers are intended to make results of ZEW research promptly available to other economists in order to encourage discussion and suggestions for revisions. The authors are solely responsible for the contents which do not necessarily represent the opinion of the ZEW. 